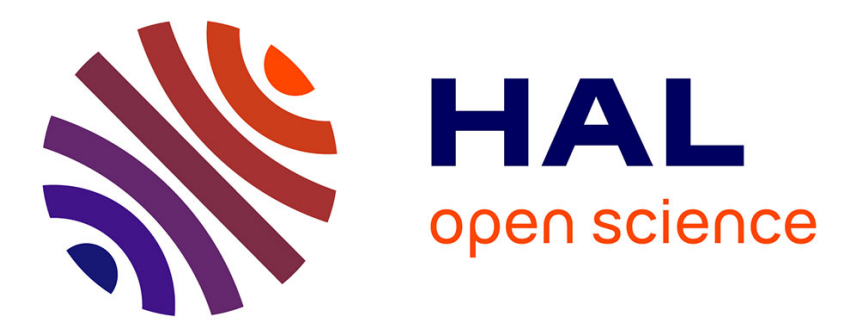

\title{
Effects of the K-value solution schemes on radiation heat transfer modelling in oxy-fuel flames using the full-spectrum correlated K-distribution method
}

\author{
Y. Liu, G. Liu, Franklin Liu, J.L. Consalvi
}

\section{- To cite this version:}

Y. Liu, G. Liu, Franklin Liu, J.L. Consalvi. Effects of the K-value solution schemes on radiation heat transfer modelling in oxy-fuel flames using the full-spectrum correlated K-distribution method. Applied Thermal Engineering, 2020, 170 (114986). hal-03189326

\section{HAL Id: hal-03189326 \\ https://hal.science/hal-03189326}

Submitted on 28 Apr 2021

HAL is a multi-disciplinary open access archive for the deposit and dissemination of scientific research documents, whether they are published or not. The documents may come from teaching and research institutions in France or abroad, or from public or private research centers.
L'archive ouverte pluridisciplinaire HAL, est destinée au dépôt et à la diffusion de documents scientifiques de niveau recherche, publiés ou non, émanant des établissements d'enseignement et de recherche français ou étrangers, des laboratoires publics ou privés. 


\title{
Effects of the K-value Solution Schemes on Radiation Heat Transfer Modelling in Oxy-
}

\section{fuel Flames Using the Full-Spectrum Correlated K-distribution Method}

\author{
Yuying LIU ${ }^{a,}$, Guanghai LIU ${ }^{a, *}$, Fengshan LIU ${ }^{b}$, Jean-louis CONSALVI \\ a. School of Energy and Power Engineering, Beihang University (BUAA), Beijing, 100191, China \\ b. Measurement Science and Standards, National Research Council of Canada, 1200 Montreal Road, Ottawa, Ontario K1A OR6, Canada \\ c. Aix-Marseille Université, CNRS, IUSTI UMR 7343, 5 rue E. Fermi, 13013 Marseille, France \\ *Corresponding author:yyliu@buaa.edu.cn(Y.LIU),ghliu@buaa.edu.cn(G.LIU)
}

\begin{abstract}
Radiation heat transfer in oxy-fuel flames is more important than in conventional fuel-air flames. The Full-Spectrum Correlated K-distribution methods (FSCK) with the original correlated-K solution scheme (Modest and Zhang, 2002) and a newly proposed one (Cai and Modest, 2014), and the Rank Correlated Full-Spectrum K-distribution method (RC-FSK) are used in radiative calculations of oxy-fuel flames. Twelve one-dimensional flames, including fuel-air, dry and wet oxy-fuel flames with various length scales, as well as a two-dimensional dry oxy-fuel flame are studied. The results show that the reference temperature has a non-negligible impact on the accuracy of original scheme and the emission-weighted temperature leads to a good accuracy. The accuracy of the new scheme is almost unaffected by the reference temperature, except for smallscale dry oxy-fuel flames. The error of the new scheme is mainly in the low-temperature region, and its accuracy depends on the length scale of computational domain. A hybrid correlated-K scheme using either the original or the new scheme according to local temperature is proposed. It combines the advantages of two schemes in different temperature regions, and is independent of the reference temperature. In addition, the RC-FSK demonstrates almost the same accuracy as FSCK with the hybrid scheme when the Planck temperature is set equal to the reference temperature.
\end{abstract}

Keywords: Full-Spectrum Correlated K-distribution; Rank Correlated Full-Spectrum K-distribution; correlated-K solution scheme; dry and wet oxy-fuel flames.

\begin{tabular}{|c|c|}
\hline Nomenclature & Subscripts \\
\hline$a \quad$ non-gray stretching factor in FSK method & $b \quad$ blackbody \\
\hline$f \quad$ full-spectrum k-distribution function & criterion \\
\hline$g \quad$ cumulative full-spectrum k-distribution function & local state \\
\hline H Heaviside step function & $m$ th direction \\
\hline$I \quad$ radiative intensity & maximum \\
\hline$k \quad$ reordered spectral absorption coefficient & $n$th quadrature point \\
\hline$L \quad$ the length of computational domain & Planck temperature \\
\hline$M \quad$ number of ordinates & reference state \\
\hline \multicolumn{2}{|l|}{$N$ number of quadrature points } \\
\hline$P \quad$ total pressure & Abbreviations \\
\hline$T$ temperature & FSCK Full-Spectrum Correlated K-distribution \\
\hline$u \quad$ direction weight & RC-FSK Rank Correlated Full-Spectrum K-distribution \\
\hline$w$ quadrature weight & LBL Line-By-Line \\
\hline \multirow[t]{2}{*}{$\underline{\boldsymbol{x}}$ mole fraction vector } & NBCK Narrow-Band Correlated K-distribution \\
\hline & SLW Spectral-Line-based Weighted-sum-of-gray-gases \\
\hline Greek symbols & SNBCK Statistical Narrow-Band Correlated-K \\
\hline $\boldsymbol{\eta} \quad$ wavenumber $\left(\mathbf{c m}^{-\mathbf{1}}\right)$ & SNB Statistical Narrow-Band \\
\hline
\end{tabular}


$\boldsymbol{\kappa}$ spectral absorption coefficient $\left(\mathbf{c m}^{-\mathbf{1}}\right)$

$\underline{\boldsymbol{\phi}}$ vector of thermodynamic state variables

$\boldsymbol{\delta}$ Dirac-delta function
WBCK Wide Band Correlated-K

WSGG Weighted-Sum-of-Gray-Gases

\section{Introduction}

Oxy-fuel combustion has drawn considerable attention in the last two decades as a promising technology for carbon capture and storage/sequestration (CCS) technology applied to reduce $\mathrm{CO}_{2}$ emissions, which is responsible for global warming [1]. Thermal radiation in oxy-fuel combustion plays a much more important role in the heat transfer process than in conventional fuel-air combustion due to the higher concentrations of recycled $\mathrm{CO}_{2}$ and/or $\mathrm{H}_{2} \mathrm{O}$ [2]. Apart from the radiative transfer equation (RTE) solution models, robust and accurate radiative property models for molecular gases, such as $\mathrm{CO}_{2}$, $\mathrm{H}_{2} \mathrm{O}$ and $\mathrm{CO}$, are also necessary for better understanding of heat transfer processes in the oxy-fuel combustion and for optimal design of oxy-fuel combustion devices [3, 4]. Radiative property models with different spectral resolutions, such as the Lineby-Line (LBL) method, narrow-band methods, wide-band methods and full-spectrum methods, demonstrate different accuracies and computational efficiencies. The "exact" LBL method, usually regarded as the benchmark, is rarely used in practical applications due to requirements in excessive computing time and resources. Narrow-band methods, such as the Statistical Narrow-Band method (SNB), the Statistical Narrow-Band Correlated-K method (SNBCK), and the Narrow-Band Correlated K-distribution method (NBCK), offer nearly the same accuracy as LBL in oxy-fuel combustion with about a thousandth of computing time and resources of LBL [5-7]. Wide-band methods, for instance, the Wide Band Correlated-K (WBCK) method, can further reduce the computing demand at loss of accuracy in oxy-fuel combustion $[8,9]$. Although the number of RTE to be solved for narrow-band and wide-band methods are much less than that of LBL, the computing resources are still very large. Therefore, the more efficient full-spectrum methods, such as the Spectral-Line-based Weighted-Sum-ofGray-Gases (SLW) method [10], the Full-Spectrum K-distribution (FSK) method [6, 7, 11, 12], and the Weighted-Sum-ofGray-Gases (WSGG) method [13-22], have been more commonly used in modelling radiative heat transfer in oxy-fuel combustion problems.

The FSK method is exact in isothermal and homogeneous media with tiny requirements in computing resources [23]. However, the error of FSK increases with increasing the temperature and/or species concentrations inhomogeneity. To deal with the inhomogeneity-induced error, several improved methods were proposed [24-26], such as the Full-Spectrum Correlated K-distribution method (FSCK) [24], the Full-Spectrum Scaled K-distribution method (FSSK) [24] and the Rank Correlated Full-Spectrum K-distribution method (RC-FSK) [25]. Owing to the correlated spectrum assumption, various correlated-k solution schemes of FSCK were developed in recent years. In the solution scheme originally proposed by Modest and Zhang [24], the Planck function temperature $T_{p}$ is replaced by the reference temperature $T_{0}$, and the correlated reordered absorption coefficient of the local state, $k^{*}$, can be directly obtained from the reference state by a one-step implementation. FSCK with this original solution scheme [24] has been widely used in both conventional fuel-air flames and oxy-fuel flames $[6,7,11,12]$. Recently, Cai and Modest [27] proposed an improved scheme to preserve the total emission, in which the Planck function temperature $T_{p}$ is replaced by the local temperature $T_{L o c}$, and $k^{*}$ can be obtained by a two-step implementation. It was reported that this improved solution scheme can effectively reduce the error of FSCK in conventional fuel-air flames, especially in the high-temperature region where the radiative source term is dominated by emission, because it ensures conservation of emission [27-29]. However, in absorption-dominated problems involving a two-layer slab with large temperature differences, as proposed by Pierrot et al. [30], the improved solution scheme does not show advantages over the original one according to Ref. [27]. More recently, in order to reduce the storage of database, Wang et al. [28] proposed a solution scheme on the basis of the Cai and Modest's scheme [27] to obtain $k^{*}$ by a three-step implementation. In this solution scheme, the Planck function temperature, $T_{p}$, is replaced by the reference temperature, $T_{0}$, in the first step, while the local temperature, $T_{L o c}$, is used as, $T_{p}$, in the third step. It was found that the FSCK methods involving either the solution schemes by Wang et al. [28] or by Cai and Modest [27] offer almost the same accuracy in conventional fuel-air flames [28]. Based on the rank correlated spectrum assumption, Solovjov et al. [25] proposed a new solution scheme, which does not require the reference state, and $k^{*}$ can be obtained directly. Comparing with conventional fuel-air flames, which are typically emission- 
dominated problems, dry and wet oxy-fuel flames are problems where emission and absorption are similarly important due to the higher concentrations of $\mathrm{CO}_{2}$ and/or $\mathrm{H}_{2} \mathrm{O}$ in low-temperature regions. Therefore, the accuracy of FSCK with the improved solution scheme of Cai and Modest [27] or other possible solution schemes in oxy-fuel flames should be further investigated before it can be used in practical engineering applications.

In addition, for the FSCK method with the original solution scheme [24] (hereafter named as FSCK-1), the reference temperature plays an important role because it is closely related to $k^{*}$. Several procedures to determine the reference temperature have been proposed and tested, such as the volume-averaged temperature [25], the Planck-mean temperature [24] and the emission-weighted temperature [24]. For conventional fuel-air flames, it was reported that FSCK-1 with the Planckmean temperature or the emission-weighted temperature as the reference temperature is much more accurate than using the volume-averaged temperature or the maximum/minimum temperature of the computational domain as the reference temperature [24]. Furthermore, FSCK-1 with the emission-weighted temperature as the reference temperature was also shown to provide a good accuracy for dry and wet oxy-fuel flames [6, 7]. In contrast, FSCK with the solution scheme by Cai and Modest [27] (hereafter referred to as FSCK-2) is almost unaffected by the reference temperature in the prediction of radiative source term of a lab-scale conventional methane-air emission-dominated flame. RC-FSK is also almost independent of the Planck temperature in the prediction of radiative source term of a 1D large-scale emission-dominated case (Example 1 of Ref [25]). Obviously, the influence of the reference temperature on FSCK-2 or the Planck temperature on RC-FSK in radiation calculation of oxy-fuel flames is worth investigating as well.

The objectives of this work are three-fold: (i) to investigate the accuracy of FSCK-1, FSCK-2 and RC-FSK in dry and wet oxy-fuel flames; (ii) to propose a new hybrid solution scheme (hereafter denoted as FSCK-3) and to investigate its accuracy in conventional fuel-air flames and dry and wet oxy-fuel flames; (iii) to investigate the effects of the reference temperature and the Planck temperature on the prediction of radiative source term and heat flux using the FSCK and RC-FSK method. Twelve one-dimensional cases, including four conventional fuel-air flames, four dry and wet oxy-fuel flames, respectively, and a two-dimensional lab-scale dry oxy-fuel flame are considered.

\section{Theoretical background}

\subsection{The Full-Spectrum Correlated K-distribution Method}

The full-spectrum k-distribution function, $f$, which is weighted by the Planck function, is defined as [24]:

$$
f_{\mathrm{T}_{P}, \underline{\phi}}(k)=\frac{1}{I_{b}\left(\mathrm{~T}_{P}\right)} \int_{0}^{\infty} I_{b \eta}\left(\mathrm{T}_{P}\right) \delta\left(k-\kappa_{\eta}(\underline{\phi})\right) \mathrm{d} \eta
$$

where $\eta$ is the wavenumber, $T_{P}$ is the Planck temperature, $I_{b \eta}$ and $I_{b}$ are respectively the spectral Planck function and the spectrally-integrated Planck function, $\delta(\cdot)$ is the Dirac-delta function, $\kappa_{\eta}$ is the spectral absorption coefficient, $\underline{\phi}$ is the vector of local state variables consisting of temperature, $T$, total pressure, $P$, and mole fraction vector, $\underline{x}$.

The cumulative full-spectrum k-distribution function, $g$, which represents the fraction of spectrum whose spectral absorption coefficient smaller than the value of $k$, is expressed as:

$g_{T_{P}, \underline{\phi}}(k)=\int_{0}^{k} f_{T_{P}, \underline{\phi}}\left(k^{\prime}\right) d k^{\prime}=\frac{1}{I_{b}\left(T_{P}\right)} \int_{0}^{\infty} I_{b \eta}\left(T_{P}\right) H\left(k-\kappa_{\eta}(\underline{\phi})\right) d \eta$

where $H(\cdot)$ is the Heaviside step function. Thus, the cumulative function $\mathrm{g}$ is a monotonically increasing function varying between 0 and 1 .

The RTE without scattering in the wavenumber-space can be converted to the reordered form in the reference g-space [24]:

$\frac{d I_{g_{0}}}{d s}=k^{*}\left(g_{0}\right)\left[a\left(g_{0}\right) I_{b}\left(T_{L o c}\right)-I_{g_{0}}\right]$

where the subscripts 0 and $L o c$ refer to the reference state and the local state, respectively. In Eq. (3), $I_{g_{0}}$ and the non-gray stretching factor, $a\left(g_{0}\right)$, are expressed as: 
$I_{g_{0}}=\frac{\int_{0}^{\infty} I_{\eta} \delta\left(k-\kappa_{\eta}\left(\underline{\phi}_{0}\right)\right) d \eta}{f_{T_{0}, \underline{\underline{x}}_{0}}(k)}$

$a\left(g_{0}\right)=\frac{f_{T_{L o c}, \underline{\underline{\phi}}_{0}}(k)}{f_{T_{0}, \underline{\underline{\phi}}_{0}}(k)}=\frac{d g_{T_{L o c}, \underline{\phi}_{0}}(k)}{d g_{T_{0}, \underline{\phi}_{0}}(k)}$

Based on the assumption that the spectral absorption coefficients of a mixture at different states are correlated, the correlated reordered absorption coefficient, $k^{*}\left(g_{0}\right)$, can be estimated by Eq. (6) [28]:

$g_{T_{P}, \underline{\phi_{0}}}(k)=g_{T_{P}, \underline{\phi_{L o c}}}\left(k^{*}\right)$

Various temperatures can be regarded as the Planck function temperature, but result in different accuracies of FSCK [24, 27, 28], and it will be discussed in different correlated-K solution schemes in the following section.

The radiative source term based on FSCK is calculated according to Eq. (7):

$$
-\nabla \cdot \dot{q}_{R}^{\prime \prime}=\int_{0}^{1}(\underbrace{4 \pi a\left(g_{0}\right) k^{*}\left(g_{0}\right) I_{b}\left(T_{L o c}\right)}_{\text {emission term }}-\underbrace{\int_{4 \pi} k^{*}\left(g_{0}\right) I_{g_{0}}}_{\text {absorption term }}) d g_{0}
$$

where $\boldsymbol{\nabla} \cdot \dot{q}_{R}^{\prime \prime}$ is the divergence of radiative flux. The radiative source term is dominated by emission in high temperature media owing to large values of the Planck function, and its accuracy is mainly dependent on both $k^{*}$ and $a$. In low temperature media, however, radiation absorption prevails and the evaluation of radiative source term depends primarily on $k^{*}$.

In the case of using the discrete ordinate method (DOM) to solve RTE, the radiative source term can be numerically obtained as follows:

$-\boldsymbol{\nabla} \cdot \dot{q}_{R}^{\prime \prime}=\sum_{n=1}^{N}\left(4 \pi a_{n} k_{n}^{*} I_{b}-\sum_{m=1}^{M} k_{n}^{*} u_{m} I_{g_{0}, n}\right) w_{n}$

where $M$ and $N$ are respectively the number of angular ordinates and quadrature points (for integration over $g$ ), $u_{m}$ and $w_{n}$ are respectively the corresponding weight of $m$ th direction and $n$th quadrature point.

\subsection{Correlated-K solution schemes}

The original correlated-K solution scheme proposed by Modest and Zhang [24] (FSCK-1), the new one proposed by Cai and Modest [27] (FSCK-2), and a hybrid scheme developed in the current work are used to evaluate the accuracies of the FSCK methods for application in oxy-fuel flames.

\subsubsection{The original correlated-K solution scheme (FSCK-1)}

For the solution scheme proposed by Modest and Zhang [24], the Planck temperature, $T_{P}$, is replaced by the reference temperature $T_{0}$; therefore, Eq. (6) can be rewritten as:

$g_{T_{0}, \underline{\phi}_{0}}(k)=g_{T_{0}, \underline{\phi_{L o c}}}\left(k^{*}\right)$

For a given $g_{0}$ value, $k^{*}$ can be evaluated by inverting Eq. (9). The top frame of Fig. 1 illustrates the $g-k$ relationship and the specific process to determine the correlated reordered absorption coefficient, $k^{*}$, for a $\mathrm{H}_{2} \mathrm{O}-\mathrm{N}_{2}$ mixture as an example. The species concentrations of both the reference and local states are set to $20 \% \mathrm{H}_{2} \mathrm{O}$ and $80 \% \mathrm{~N}_{2}$, and the reference and local temperatures are $1000 \mathrm{~K}$ and $1500 \mathrm{~K}$, respectively. For an arbitrary $g_{0}$ value (here $g_{0}=0.2$ ), $k^{*}$ can be directly obtained from point A to point B by a one-step procedure. The total emission term of the radiative source term (as shown in Eq. (7)) evaluated through this procedure is given as [27-29]:

$\int_{0}^{1} k^{*}\left(g_{0}\right) \cdot a\left(g_{0}\right) d g_{0}$

$=\int_{0}^{1} k_{T_{0}, \underline{\phi}_{L o c}}\left(g_{T_{0}, \underline{\phi}_{0}}\right) \cdot \frac{d g_{T_{L o c}, \underline{\phi}_{0}}}{d g_{T_{0}, \underline{\phi}_{0}}} d g_{T_{0}, \underline{\phi}_{0}}$

$=\int_{0}^{1} k_{T_{0}, \underline{\phi_{L o c}}}\left(g_{T_{0}, \underline{\phi}_{0}}\right) \cdot d g_{T_{L o c}, \underline{\phi}_{0}}$

$\approx \int_{0}^{1} k_{T_{L o c}, \underline{\phi_{L o c}}}(g) \cdot d g$ 
where $\kappa_{P}$ is the Planck-mean absorption coefficient. In other words, the error of the total emission term appears and increases with increasing the inhomogeneity in temperature and/or species concentration, because the correlated spectrum assumption becomes increasingly less valid.

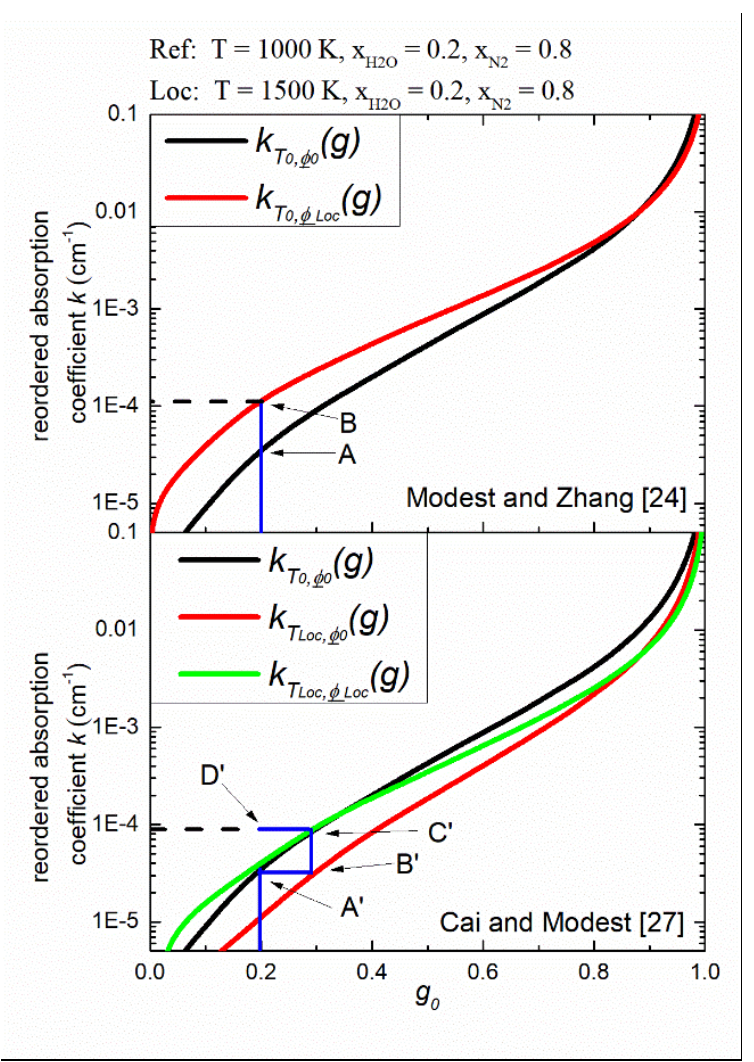

Fig. 1 Schematic diagram of the FSCK-1 and FSCK-2 implementations to determine the correlated reordered absorption coefficient $k^{*}$. The reference state: $20 \% \mathrm{H}_{2} \mathrm{O}, 80 \% \mathrm{~N}_{2}$ and $1000 \mathrm{~K}$ and the local state: $20 \% \mathrm{H}_{2} \mathrm{O}, 80 \% \mathrm{~N}_{2}$ and $1500 \mathrm{~K}$.

\subsubsection{The improved correlated-K solution scheme (FSCK-2)}

In the improved correlated-K solution scheme proposed by Cai and Modest [27], the Planck function temperature, $T_{P}$, is replaced by the local temperature $T_{L o c}$, and Eq. (6) can be rewritten as:

$g_{T_{L o c}, \underline{\phi}_{0}}(k)=g_{T_{L o c}, \underline{\phi_{L o c}}}\left(k^{*}\right)$

For a given $g_{0}$ value, $k^{*}$ can be obtained through a two-step procedure:

$k_{T_{0}, \underline{\phi}_{0}}\left(g_{0}\right) \underset{1 s t}{\longrightarrow} g_{T_{L o c}, \underline{\phi}_{0}}\left(k_{T_{0}, \underline{\phi}_{0}}\right) \underset{2 n d}{\longrightarrow} k_{T_{L o c}, \underline{\phi_{L o c}}}\left(g_{T_{L o c}, \underline{\phi}_{0}}\right)$

The bottom frame of Fig. 1 illustrates this procedure and shows the three related $g-k$ distributions, namely $k_{T_{0}}, \underline{\phi}_{0}(g)$, $k_{T_{L o c}, \underline{\phi}_{0}}(g)$ and $k_{T_{L o c}, \underline{\phi}_{L o c}}(g)$. For a given $g_{0}$ value (here $\left.g_{0}=0.2\right)$, the reordered absorption coefficient at the reference temperature and reference state, $k_{T_{0}, \underline{\phi}_{0}}\left(g_{0}\right)$ (point $\mathrm{A}^{\prime}$ ), can be obtained. Then, the corresponding $\mathrm{g}$ value (point B') is obtained through the first step of the procedure in Eq. (12). The reordered absorption coefficients at point A and point B' are identical, because they share the same absorption coefficients at the reference state. Like the approach of Modest and Zhang [24], $k_{T_{L o c}, \underline{\phi}_{L o c}}^{*}$ (point $C^{\prime}$ ) can be evaluated by inverting Eq. (11), i.e., the second step of the procedure expressed in Eq. (12). 
Finally, $k^{*}$ can be mapped into reference g-space, i.e., point D'. The total emission term evaluated through this method is given by Eq. (13)

$\int_{0}^{1} k^{*}\left(g_{0}\right) \cdot a\left(g_{0}\right) d g_{0}=\kappa_{P}$

i.e., the improved solution scheme ensures the conservation of emission [27-29].

\subsubsection{The hybrid correlated-K solution scheme (FSCK-3)}

Figure 2 compares the $k^{*}$ profiles obtained by the original [24] and the improved solution scheme [27] for five local temperatures between 300 and $2000 \mathrm{~K}$ at a reference temperature of $1000 \mathrm{~K}$. Both the reference and local states are composed of $20 \% \mathrm{H}_{2} \mathrm{O}$ and $80 \% \mathrm{~N}_{2}$. When the reference temperature equals to the local temperature, the profile of $k^{*}$ of the improved solution scheme [27] is identical to that of the original one [24] (the solid blue line), which can be easily explained by comparing Eq. (9) with Eq. (11). The discrepancy between the two $k^{*}$ profiles of FSCK-1 and FSCK-2 increases with increasing the difference between the reference and local temperatures. In the high temperature regions, the FSCK-2 scheme is expected to be more accurate than the FSCK-1 since it preserves total emission. On the other hand, numerical experiences revealed that, in the low temperature regions, the FSCK-1 is more accurate than the FSCK-2. As a consequence, a hybrid solution scheme is proposed and tested here, which uses either the FSCK-1 or the FSCK-2 according to the local temperature. The reference temperature is taken as the criterion, i.e., the FSCK-2 solution scheme of Cai and Modest [27] is applied when the local temperature is higher than the reference temperature; otherwise, the FSCK-1 solution scheme of Modest and Zhang [24] is used. The aim of this hybrid solution scheme of FSCK proposed here is to combine the advantages of FSCK-1 and FSCK-2 in the low- and high-temperature regions, respectively. The influence of the criterion on the prediction will be discussed later.

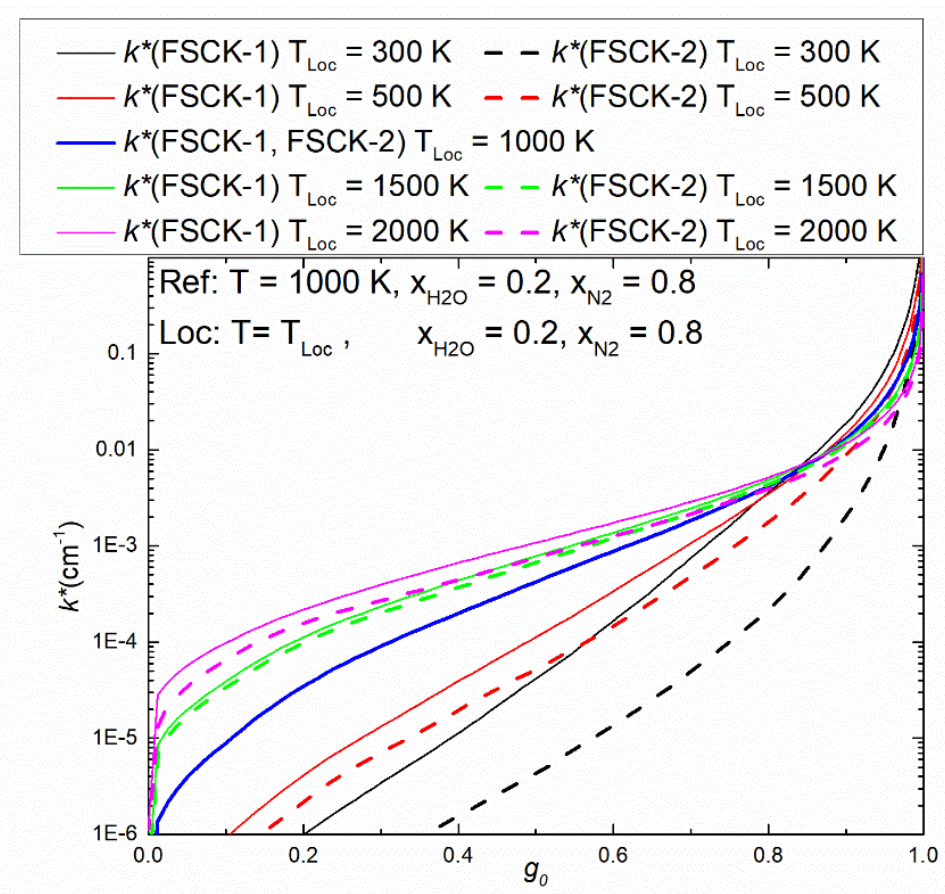

Fig. 2 The profiles of $k^{*}$ evaluated by FSCK-1 and FSCK-2 at five local temperatures of 300, 500, 1000, 1500, and $2000 \mathrm{~K}$. The reference state is $20 \% \mathrm{H}_{2} \mathrm{O}, 80 \% \mathrm{~N}_{2}$ and $1000 \mathrm{~K}$. The local state has the same species concentrations as the reference state.

\subsection{The Rank Correlated Full-Spectrum K-distribution method}

There are a few differences between the FSCK method and the RC-FSK method. Based on the rank correlated spectrum assumption, the spectral absorption coefficients at any two states are correlated. Therefore, the RC-FSK method does not require specifying a reference state [25]. The RTE of RC-FSK is identical to that of FSCK. It solves $g_{0}=g_{T_{p}, \underline{\phi}_{L o c}}\left(k^{*}\right)$ to 
get $k^{*}$ and computes $g_{T_{L o c}, \underline{\phi_{L o c}}}\left(k^{*}\right)$ to determine the non-gray stretching factor, $a$, according to Eq. (14)

$a\left(g_{0}\right)=\frac{d g_{T_{L o c}, \underline{\underline{\underline{\phi}}} L o c}\left(k_{T p, \underline{\underline{\phi}} L o c}\left(g_{0}\right)\right)}{d g_{0}}$

It should be noted that the subscript 0 in Eq. (14) here does not refer to a reference state. It is used here solely for the purpose of retaining the same form as the FSCK model given in Eqs. (3) - (5).

It is noticed that in RC-FSK the Planck temperature $T_{P}$ is still a model parameter. Figure 3 illustrates the specific process to determine $k^{*}$ of FSCK-1 and RC-FSK. In this example, the Planck temperature (RC-FSK) and the reference temperature (FSCK-1) are set at $1000 \mathrm{~K}$. Only one $g-k$ distributions, namely $k_{T_{P}, \phi_{L o c}}(g)$, is used to obtain $k^{*}$ for RC-FSK. For instance, for an arbitrary $g_{0}$ value (here $g_{0}=0.2$ ), $k^{*}$ can be directly obtained (point A"). The total emission term evaluated through this procedure is given as [25]:

$\int_{0}^{1} k^{*}\left(g_{0}\right) \cdot a\left(g_{0}\right) d g_{0}=\kappa_{P}$

i.e., the RC-FSK method also preserves the conservation of emission as FSCK-2, which implies that RC-FSK and FSCK-2 are likely to provide similar results in high-temperature emission-dominated regions. Furthermore, it can be seen from Fig. 3 that FSCK-1 and RC-FSK share the identical $k^{*}$ when the reference temperature for FSCK-1 is equal to the Planck temperature for RC-FSK, which suggests that RC-FSK and FSCK-1 yield similar results in low-temperature absorption-dominated regions (see Eq. (7)). In conclusion, RC-FSK may offer the similar accuracy as the FSCK-3 method.

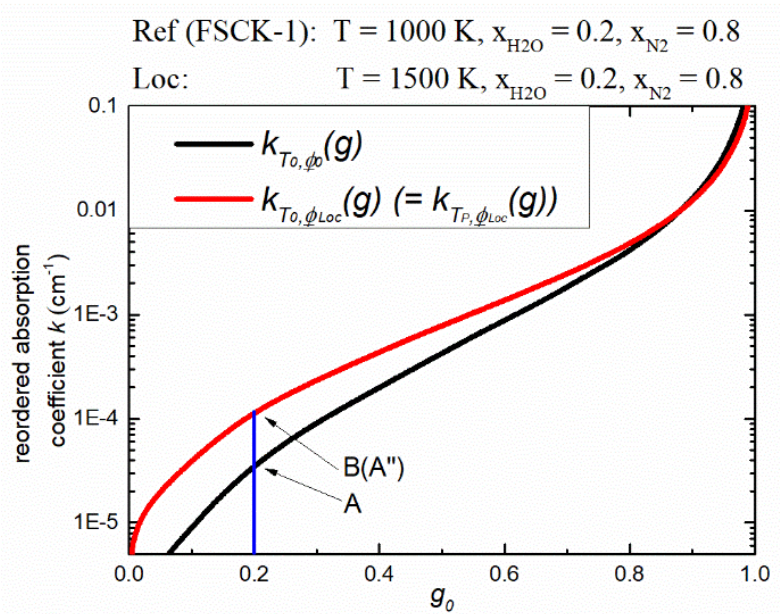

Fig. 3 Schematic diagram of the FSCK-1 and RC-FSK implementations to determine the correlated reordered absorption coefficient $k^{*}$. The reference state (for FSCK-1 only): $20 \% \mathrm{H}_{2} \mathrm{O}, 80 \% \mathrm{~N}_{2}$ and $1000 \mathrm{~K}$ and the local state: $20 \% \mathrm{H}_{2} \mathrm{O}, 80 \% \mathrm{~N}_{2}$ and $1500 \mathrm{~K}$. In this special case of $T_{\mathrm{p}}=T_{0}$, RC-FSK leads to identical $k^{*}$ as FSCK-1.

\section{Results and discussion of one-dimensional flames}

Calculations of radiative heat transfer in various 1D counter-flow flames, including four conventional fuel-air flames (Case 1), four dry (Case 2) and four wet (Case 3) oxy-fuel flames, with different separation distances are conducted to investigate the accuracies of FSCK-1, FSCK-2, FSCK-3 and RC-FSK. The oxy-fuel flames are based on the fuel-air counterflow diffusion flame of Cheng et al. [31] with the separation distance of $L=0.0126 \mathrm{~m}$. The dry oxy-fuel flames are constructed from Case 1 by uniformly adding 0.3 to the mole fraction of $\mathrm{CO}_{2}$, whereas the temperature and water vapour mole fraction profiles are kept unchanged. The wet oxy-fuel flames are also constructed from Case 1 by adding 0.3 uniformly to both the mole fractions of $\mathrm{CO}_{2}$ and $\mathrm{H}_{2} \mathrm{O}$. For each case, larger scale flames with separation distances of $L=0.126 \mathrm{~m}, 0.63 \mathrm{~m}$ and 1.26 $\mathrm{m}$ are also constructed to achieve larger optical thickness. The test cases are summarized in Table 1. It is assumed that the two bounding walls at the position of the opposed jets are black and at $300 \mathrm{~K}$. 
Table 1. Descriptions of the thermal conditions of the twelve one-dimensional test cases.

\begin{tabular}{|c|c|c|c|c|c|}
\hline Case & Sub-case & $\begin{array}{c}\text { Separation } \\
\text { distance } L(\mathrm{~m})\end{array}$ & $\begin{array}{c}\text { Temperature/ } \\
\mathrm{x}_{\mathrm{CO} 2} / \mathrm{x}_{\mathrm{H} 2 \mathrm{O}} \\
\end{array}$ & Flame type & Notes \\
\hline \multirow{4}{*}{1} & $\mathrm{a}$ & 0.0126 & \multirow{4}{*}{ See Fig. 4} & \multirow{4}{*}{$\begin{array}{l}\text { Conventional } \\
\text { fuel-air flames }\end{array}$} & $\operatorname{Ref}[31]$ \\
\hline & $\mathrm{b}$ & 0.126 & & & \\
\hline & $\mathrm{c}$ & 0.63 & & & \\
\hline & d & 1.26 & & & \\
\hline \multirow{4}{*}{2} & $\mathrm{a}$ & 0.0126 & \multirow{4}{*}{ See Fig. 7} & \multirow{4}{*}{ dry oxy-fuel flames } & \\
\hline & $\mathrm{b}$ & 0.126 & & & \\
\hline & $\mathrm{c}$ & 0.63 & & & \\
\hline & d & 1.26 & & & \\
\hline \multirow{4}{*}{3} & $\mathrm{a}$ & 0.0126 & \multirow{4}{*}{ See Fig. 11} & \multirow{4}{*}{ wet oxy-fuel flames } & \\
\hline & $\mathrm{b}$ & 0.126 & & & \\
\hline & $\mathrm{c}$ & 0.63 & & & \\
\hline & $\mathrm{d}$ & 1.26 & & & \\
\hline
\end{tabular}

DOM along with the S8 angular discretization scheme [32] is used to solve RTE. The 32-point Gauss-Chebyshev scheme is chosen as the quadrature scheme in calculations of both FSCK and RC-FSK. The emission-weighted temperature is selected as the reference temperature for FSCK-1, FSCK-2 and FSCK-3, and as the Planck temperature for RC-FSK in all the flames. The influence of the reference temperature and Planck temperature on the accuracy of these models will be discussed later. The spectral absorption coefficients of radiating media are derived from the HITEMP 2010 database [33] with the HAPI code [34]. The wavenumber ranges from $200 \mathrm{~cm}^{-1}$ to $10000 \mathrm{~cm}^{-1}$ and the wavenumber interval is set to $0.02 \mathrm{~cm}^{-1}$ in LBL calculations, which was found to be a good compromise between accuracy and efficiency [35].

The radiative source term and corresponding local relative error (LRE) benchmarked by the LBL solution according to Eq. (16) are discussed for all the flames. The radiative heat flux distribution along the separation position is presented as well.

$\operatorname{LRE}(\mathrm{x})=\frac{-\nabla \cdot \dot{q}_{R}^{\prime \prime}{ }_{F S C K}(x)+\nabla \cdot \dot{q}_{R L B L}^{\prime \prime}(x)}{\left|\nabla \cdot \dot{q}_{R L L}^{\prime \prime}(x)\right|_{\max }} \times 100 \%$

For the convenience of discussion, the computational domain of all cases are roughly divided into two regions, namely the high-temperature region where the temperature is higher than $1000 \mathrm{~K}$, and the low-temperature region in which the temperature is lower than $1000 \mathrm{~K}$, as shown in Figure 1.

\subsection{Conventional fuel-air flames}

Figure 4 shows the distributions of temperature and mole fractions of $\mathrm{CO}_{2}$ and $\mathrm{H}_{2} \mathrm{O}$ of Case 1 normalized by the separation distance $L$. It can be seen that the radiative participating species are mainly concentrated in the high-temperature region, while the mole fractions of $\mathrm{CO}_{2}$ and $\mathrm{H}_{2} \mathrm{O}$ are much less in the low-temperature region (actually drops to zero at the fuel and oxidizer inlets) in the conventional fuel-air diffusion flame. 


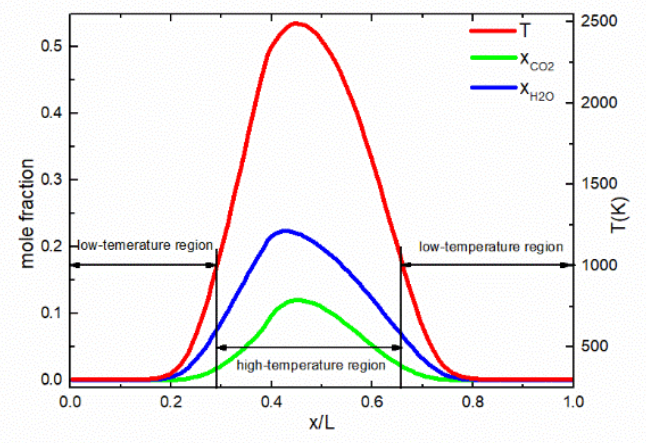

Fig. 4 Distributions of temperature and mole fractions of $\mathrm{CO}_{2}$ and $\mathrm{H}_{2} \mathrm{O}$ for Case 1 .

Figure 5 shows the radiative source terms and their local relative errors, LREs, of the four conventional fuel-air flames. It can be seen that the predictions by FSCK-1, FSCK-2, FSCK-3 and RC-FSK are in good agreement with the benchmark LBL solution. FSCK-1 demonstrates good accuracy when the emission-weighted temperature is used, as reported in Refs. [24, $32,36]$. FSCK-2 and RC-FSK can accurately predict the emission term of the source term, as shown in Eq. (13) and (15) [25, 27-29], which explains their accuracy. It should be pointed out that the absorption term in the conventional fuel-air flames is negligible (in the high-temperature region, the absorption term is much smaller than the emission term, while the concentrations of radiating species in the low-temperature regions are very low). In addition, for each separation distance, the radiative source term predicted by FSCK-3 is almost undistinguishable as compared to the benchmark as well, i.e., FSCK-3 can be applied to radiative calculations of conventional fuel-air flames.

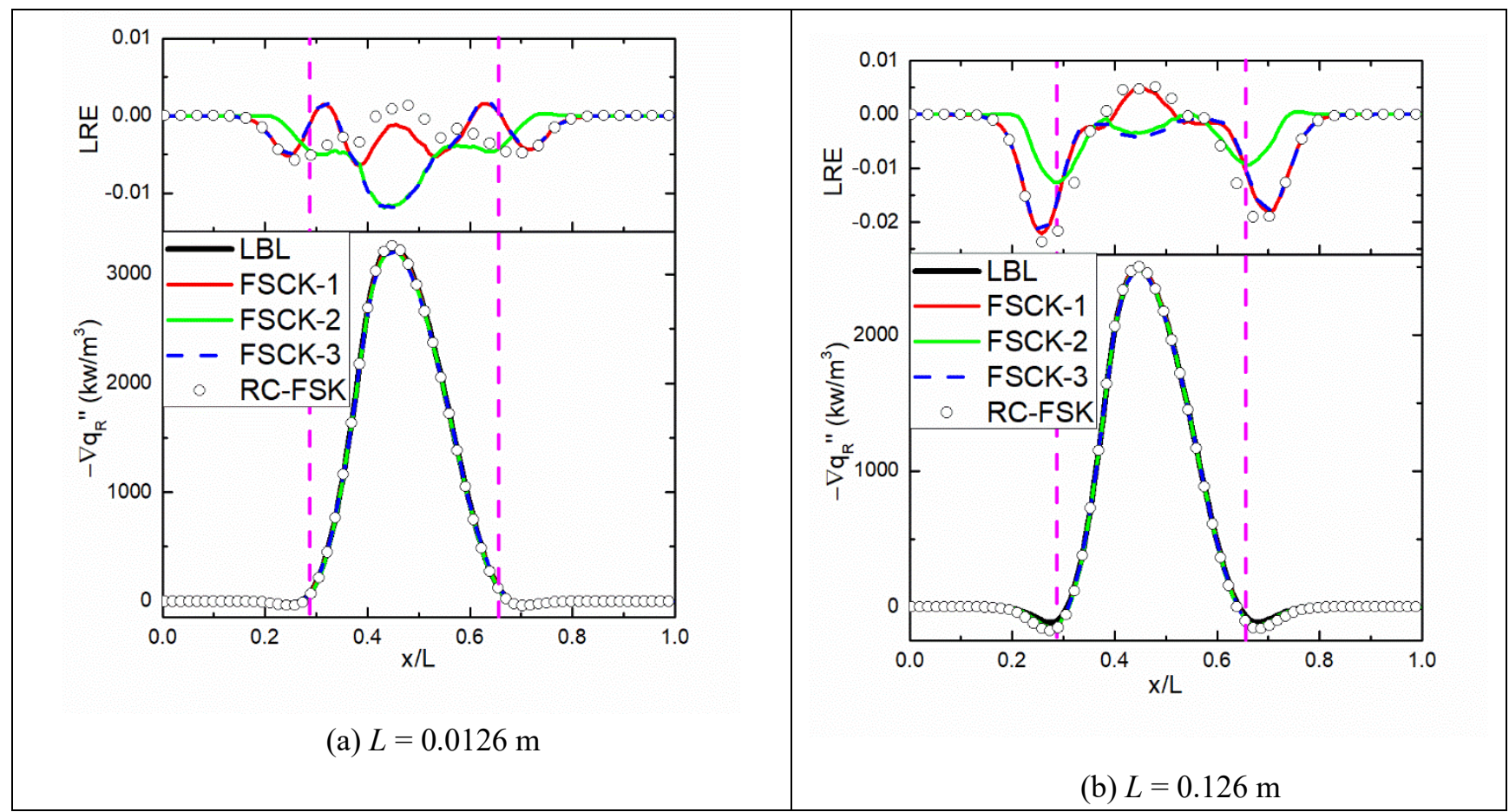




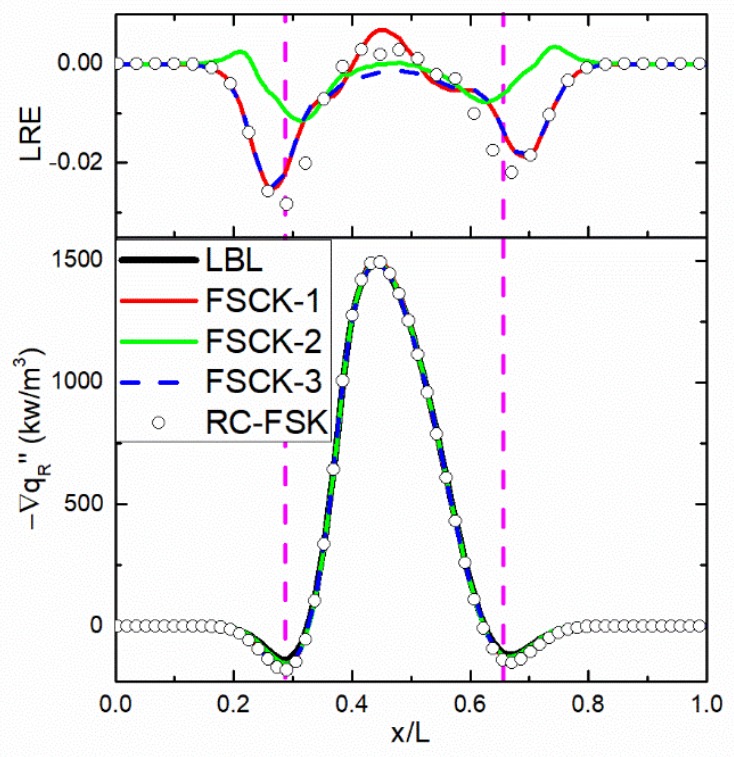

(c) $L=0.63 \mathrm{~m}$

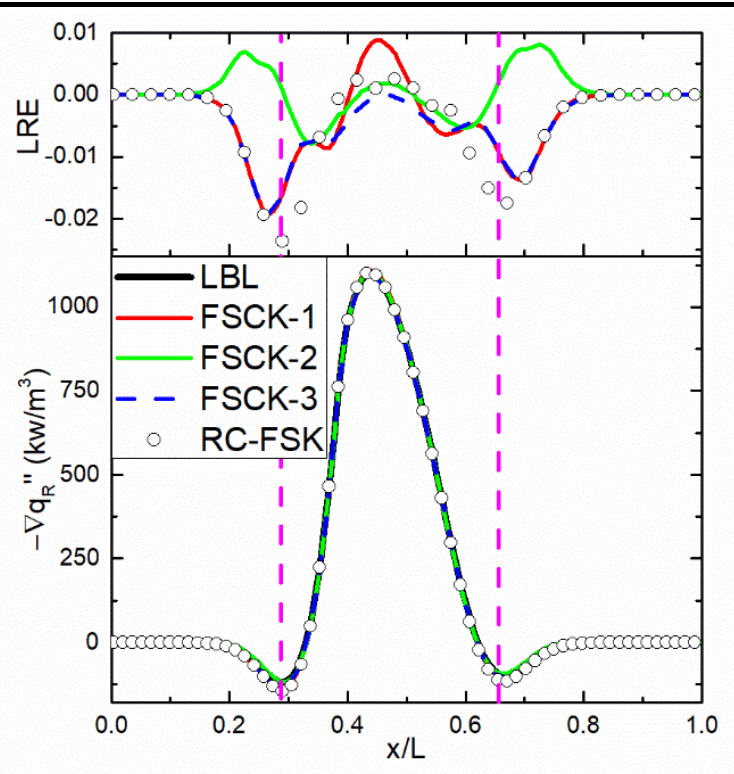

(d) $L=1.26 \mathrm{~m}$

Fig. 5 Distributions of the predicted radiative source terms and local relative errors (LREs) by FSCK-1, FSCK-2, RC-FSK, FSCK-3, and LBL in the four conventional fuel-air flames of different separation distances (Case 1). For each diagram, the bottom frame shows the distributions of the radiative source term and the top frame shows the local relative errors benchmarked by the result of LBL solution.

Figure 6 displays the radiative heat flux distributions predicted by FSCK-1, FSCK-2, FSCK-3, RC-FSK and LBL. The results of FSCK-1, FSCK-2, FSCK-3 and RC-FSK are all in very good agreement with the benchmark LBL solutions, regardless of the separation distance, which is consistent with the results shown in Fig. 5.

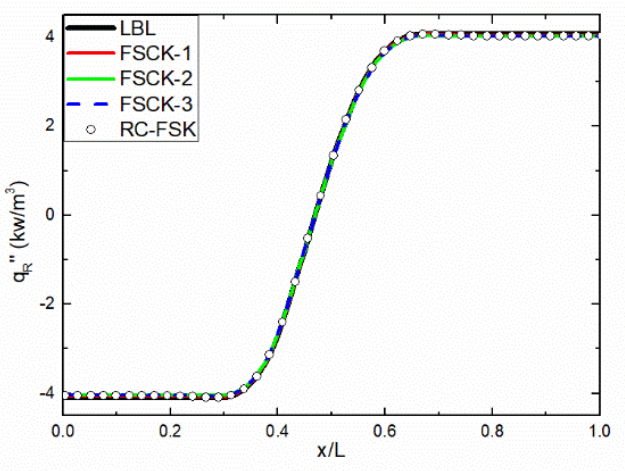

(a) $L=0.0126 \mathrm{~m}$

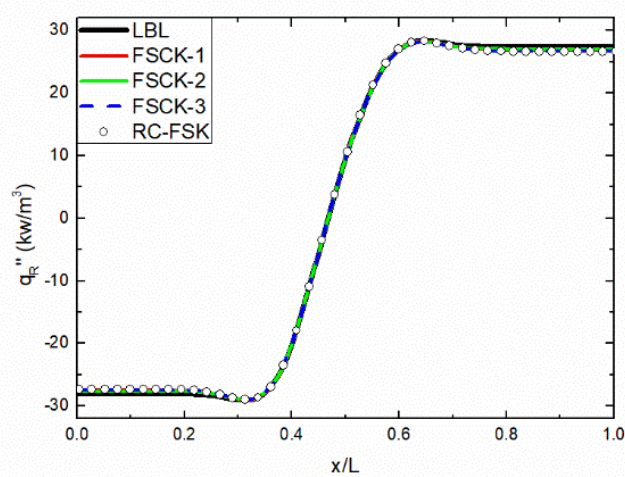

(b) $L=0.126 \mathrm{~m}$ 


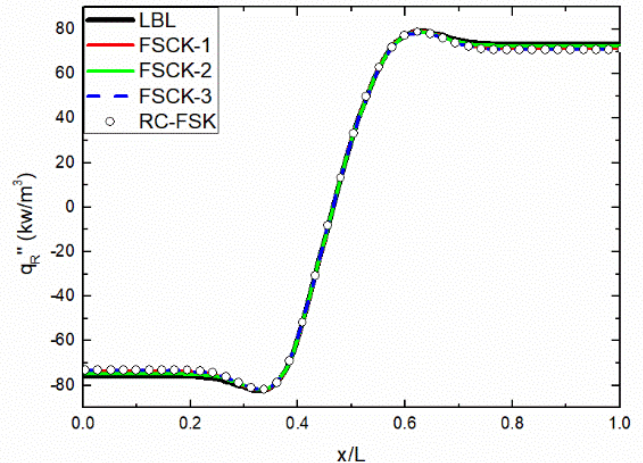

(c) $L=0.63 \mathrm{~m}$

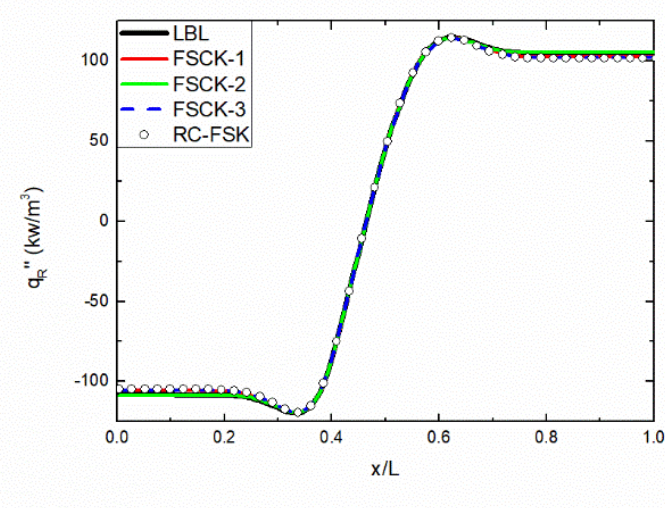

(d) $L=1.26 \mathrm{~m}$

Fig. 6 Distributions of the radiative heat flux predicted by FSCK-1, FSCK-2, RC-FSK, FSCK-3, and LBL in the four conventional fuel-air flames of different separation distances (Case 1).

\subsection{Dry oxy-fuel flames}

Four dry oxy-fuel flames with the same thermal conditions but different separation distances are included in Case 2. Figure 7 displays the distributions of temperature and mole fractions of $\mathrm{CO}_{2}$ and $\mathrm{H}_{2} \mathrm{O}$ for Case 2, which are the same as those of the conventional fuel-air flames shown in Fig. 4, except the $\mathrm{CO}_{2}$ mole fraction is increased by 0.3 uniformly. This leads to a much higher concentration of $\mathrm{CO}_{2}$ compared to Case 1, especially in the low-temperature region. As such, absorption in these regions is expected to be important.

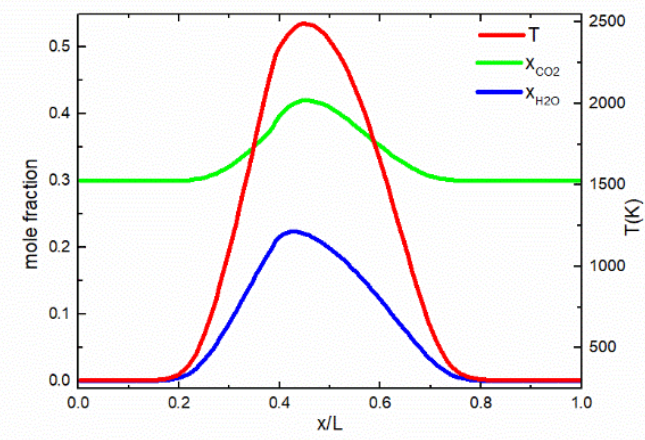

Fig. 7 Distributions of temperature and mole fractions of $\mathrm{CO}_{2}$ and $\mathrm{H}_{2} \mathrm{O}$ for Case 2.

Figure 8 and Table 2 show the radiative source terms and corresponding maximum LREs for the four dry oxy-fuel flames. Similar to its performance in Case 1, FSCK-1 can provide good accuracy in comparison with the benchmark, which is consistent with the conclusions of Kez et al. [6,7]. To be more specific, the prediction in the high-temperature region is in very good agreement with the LBL solution due to appropriate selection of the reference temperature; however, in the lowtemperature region, higher discrepancies between the FSCK-1 predictions and LBL can be observed. In contrast, FSCK-2 demonstrates the same accuracy as FSCK-1 in the high-temperature region due to the conservation of emission. However, in the low-temperature region, LREs of FSCK-2 is much larger than that of FSCK-1, especially for two small-scale flames, i.e., Case $2 \mathrm{a}$ and $2 \mathrm{~b}$. For example, the maximum LREs in the low-temperature region of Case $2 \mathrm{a}$ are $-8.5 \%$ by FSCK-1 and $-33.1 \%$ by FSCK-2.

Table 2. The maximum local relative errors of the predicted radiative source term for Case 2.

\begin{tabular}{|c|c|c|c|c|c|c|c|c|}
\hline \multirow{2}{*}{ Case } & \multicolumn{4}{|c|}{ High-temperature region (\%) } & \multicolumn{4}{c|}{ Low-temperature region (\%) } \\
\cline { 2 - 8 } & FSCK-1 & FSCK-2 & RC-FSK & FSCK-3 & FSCK-1 & FSCK-2 & RC-FSK & FSCK-3 \\
\hline
\end{tabular}




\begin{tabular}{|c|c|c|c|c|c|c|c|c|}
\hline $2 \mathrm{a}$ & -1.6 & -1.6 & -2.5 & -1.4 & -8.5 & $\mathbf{- 3 3 . 1}$ & -8.7 & -8.3 \\
\hline $2 \mathrm{~b}$ & -3.0 & -3.0 & -3.8 & -3.0 & -4.2 & $\mathbf{- 2 1 . 2}$ & -4.5 & -4.2 \\
\hline $2 \mathrm{c}$ & 1.1 & -1.1 & -2.4 & -1.2 & -2.1 & -3.5 & -2.7 & -2.0 \\
\hline $2 \mathrm{~d}$ & 1.5 & 2.1 & -1.8 & -1.2 & -0.7 & 1.6 & -0.7 & -0.7 \\
\hline
\end{tabular}

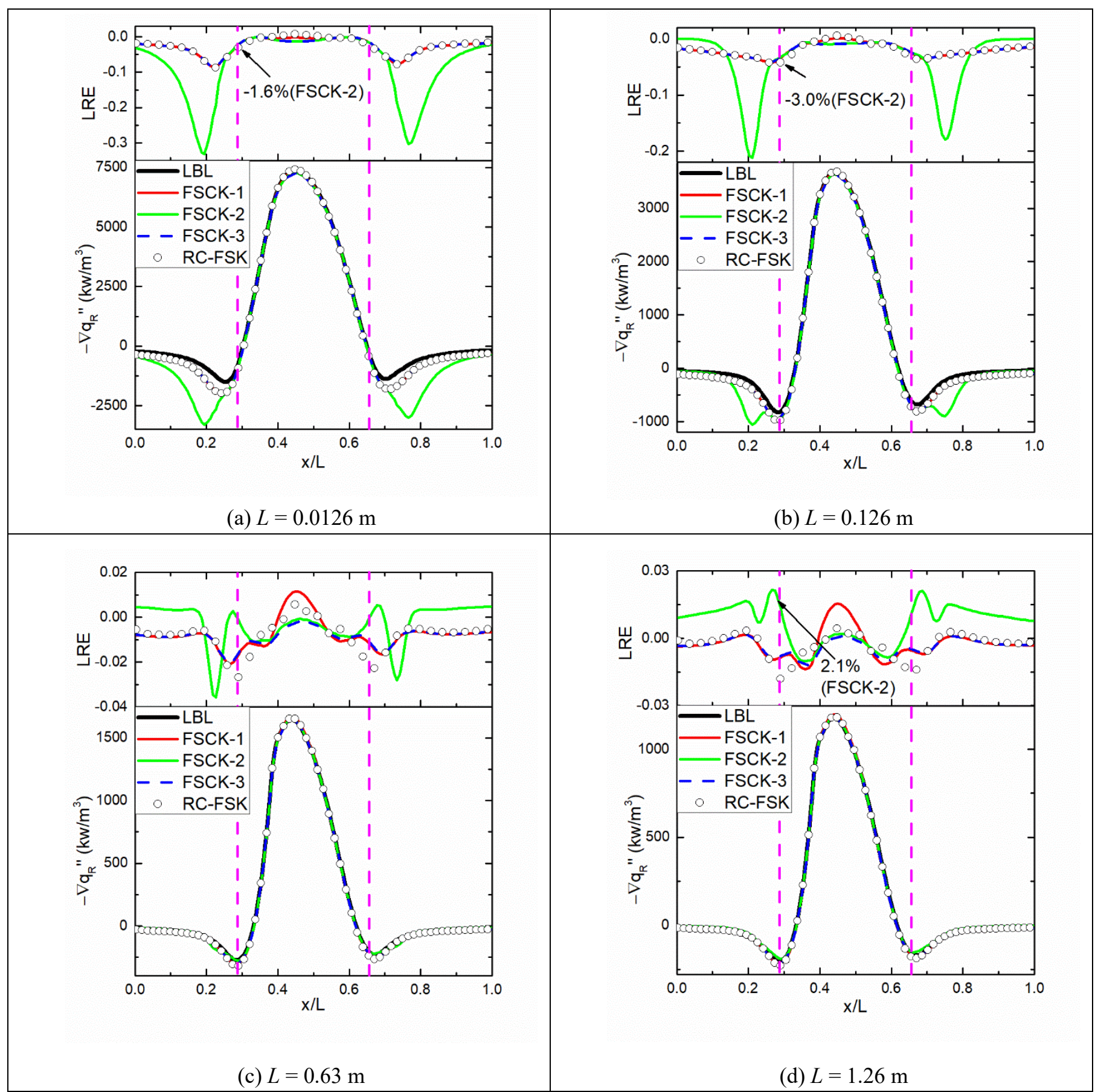

Fig. 8 Distributions of the predicted radiative source terms and local relative errors (LREs) by FSCK-1, FSCK-2, RC-FSK, FSCK-3, and LBL in the four dry oxy-fuel flames of different separation distances (Case 2). For each diagram, the bottom frame shows the distributions of the radiative source term and the top frame shows the local relative errors benchmarked by the result of LBL.

To help understand the poorer performance of FSCK-2 than FSCK-1 in the low-temperature region of Case 2, especially Case 2a, Figure 9 shows the profiles of $k^{*}$ at two positions in the low-temperature region according to the solution schemes 
by Modest and Zhang (FSCK-1) [24] and Cai and Modest (FSCK-2) [27]. It can be seen that the $k^{*}$ profiles obtained with these two solution schemes are very different. For instance, considering the $k^{*}$ value at $g_{0}=0.6$ for the local state $1\left(T_{L o c}=\right.$ $306 \mathrm{~K}, x_{\mathrm{CO}_{2}}=3.0 \times 10^{-1}, x_{\mathrm{H}_{2} \mathrm{O}}=7.0 \times 10^{-4}$ ), they are $2.6 \times 10^{-6} \mathrm{~cm}^{-1}$ and $1.5 \times 10^{-7} \mathrm{~cm}^{-1}$ by FSCK-1 and FSCK-2, respectively. As a consequence, the large discrepancy between $k^{*}$ in FSCK-2 and FSCK-1 explains the larger errors of FSCK-2. The large errors of FSCK-2 in Case 2a may possibly be due to the following two reasons. First, with decreasing the optical thickness (or the separation distance), the local absorption of thermal emissions from the high-temperature region plays an increasing role to the radiative source term in the low-temperature regions. Second, $\mathrm{CO}_{2}$ is the only absorbing species in the low-temperature regions of Case 2 and the absorption coefficient of $\mathrm{CO}_{2}\left(k^{*}\right)$ displays a strong decrease with decreasing the local temperature in FSCK-2, which uses the local temperature as the Planck temperature. The latter point can be clearly observed in Fig. 9, especially at state 1. Therefore, the significantly under-predicted absorption coefficient of $\mathrm{CO}_{2}$ by FSCK2 in the low-temperature region causes the large errors.

The predictions of RC-FSK and FSCK-3 (the blue dotted line of Fig. 8) agree well with that of LBL. The radiative source terms by RC-FSK and FSCK-3 are in very close agreement with those of FSCK-2 and FSCK-1 in the high- temperature region, regardless the reference temperature (for FSCK-1, FSCK-2 and FSCK-3) and Planck temperature (for RC-FSK), which will be discussed later.

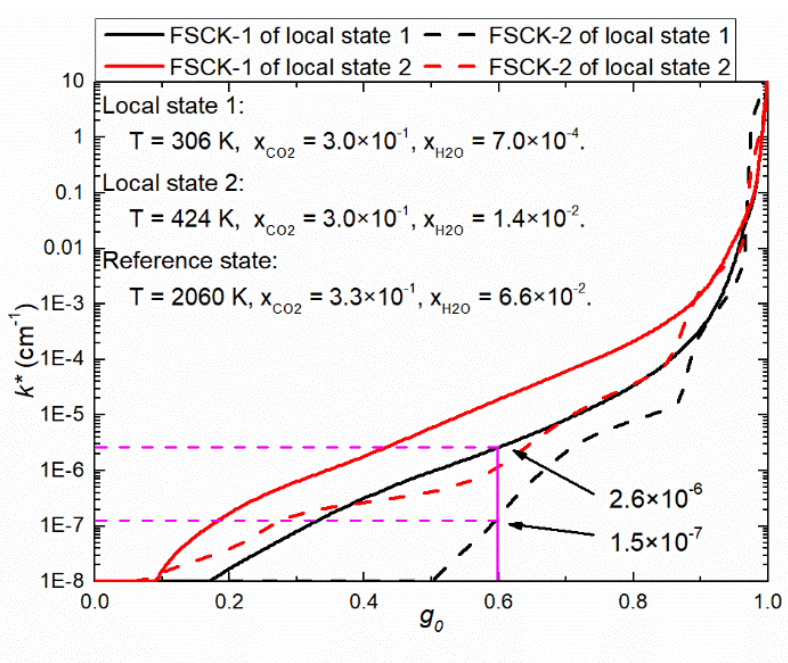

Fig. 9 The profiles of $k^{*}$ at two local states of low-temperature region of Case 2 calculated by FSCK-1 and FSCK-2.

(The reference state: $\mathrm{T}=2060 \mathrm{~K}, x_{\mathrm{CO}_{2}}=3.3 \times 10^{-1}, x_{\mathrm{H}_{2} \mathrm{O}}=6.6 \times 10^{-2}$;

local state 1: $\mathrm{T}=306 \mathrm{~K}, x_{\mathrm{CO}_{2}}=3.0 \times 10^{-1}, x_{\mathrm{H}_{2} \mathrm{O}}=7.0 \times 10^{-4}$;

local state $\left.2: \mathrm{T}=424 \mathrm{~K}, x_{\mathrm{CO}_{2}}=3.0 \times 10^{-1}, x_{\mathrm{H}_{2} \mathrm{O}}=1.4 \times 10^{-2}\right)$.

Figure 10 displays the radiative heat flux distributions predicted by FSCK-1, FSCK-2, FSCK-3, RC-FSK and LBL for the dry oxy-fuel flames (Case 2), which are different from the emission-dominated flames in Case 1. For small-scale dry oxyfuel flames (Case 2a and 2b), the results of FSCK-2 are significantly less accurate than those of FSCK-1, FSCK-3 and RCFSK, while FSCK-1, FSCK-3 and RC-FSK demonstrate very similar accuracy. For Case 2c, FSCK-1, FSCK-2, FSCK-3 and RC-FSK are in close agreement with the benchmark LBL solution. For Case 2d, FSCK-1, FSCK-3 and RC-FSK provide good accuracy, while the error of FSCK-2 rises again. In other words, FSCK-1, FSCK-3 and RC-FSK display consistently good performance in Case 2, regardless of the separation distance, while the separation distance has a great impact on the accuracy of FSCK-2. 


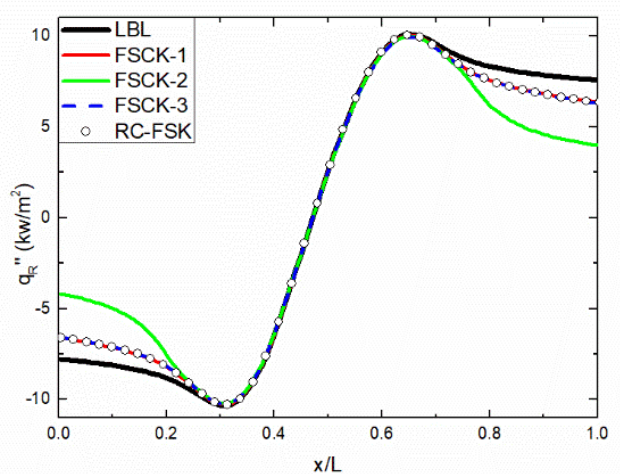

(a) $L=0.0126 \mathrm{~m}$

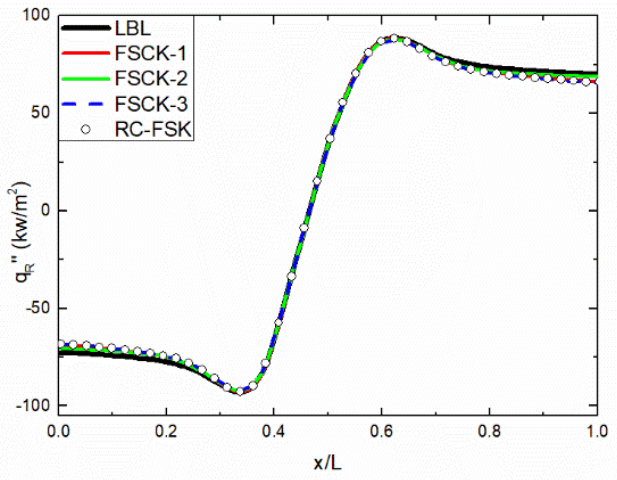

(c) $L=0.63 \mathrm{~m}$

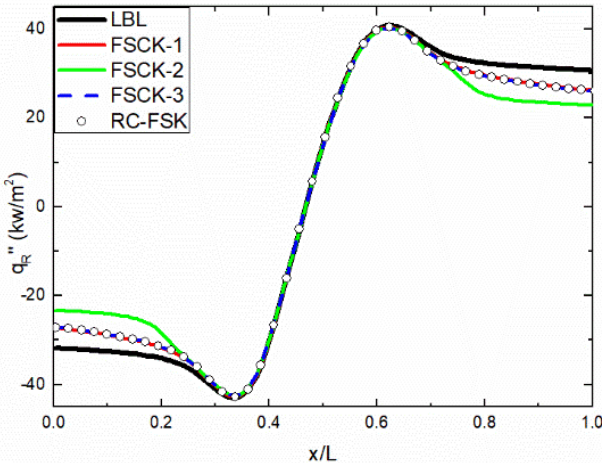

(b) $L=0.126 \mathrm{~m}$

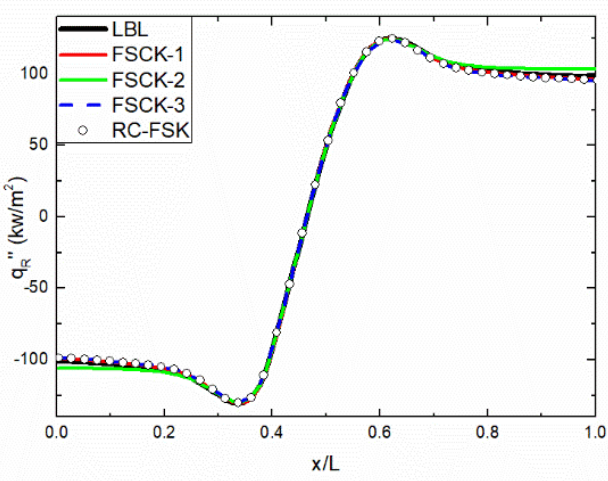

(d) $L=1.26 \mathrm{~m}$

Fig. 10 Distributions of the radiative heat flux predicted by FSCK-1, FSCK-2, RC-FSK, FSCK-3, and LBL in the four dry oxy-fuel flames of different separation distances (Case 2).

\subsection{Wet oxy-fuel flames}

Case 3 is composed of four wet oxy-fuel flames. Figure 11 displays the profiles of temperature and mole fractions of $\mathrm{CO}_{2}$ and $\mathrm{H}_{2} \mathrm{O}$ for Case 3, which are the same as those of the dry oxy-fuel flames shown in Fig. 7, except that the mole fraction of $\mathrm{H}_{2} \mathrm{O}$ is raised everywhere by 0.3 . Similar to the dry oxy-fuel flames, the wet oxy-fuel flames are also such radiative heat transfer problems where absorption can be important due to the elevated concentrations of $\mathrm{CO}_{2}$ and $\mathrm{H}_{2} \mathrm{O}$ in the lowtemperature regions.

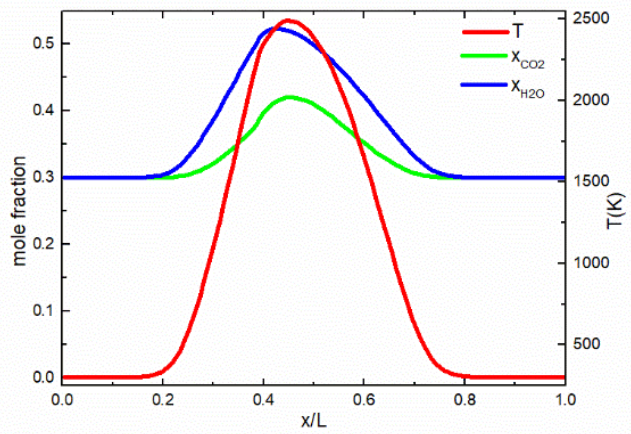

Fig. 11 Distributions of temperature and mole fractions of $\mathrm{CO}_{2}$ and $\mathrm{H}_{2} \mathrm{O}$ of Case 3 . 
The radiative source terms calculated by the four approximate models (FSCK-1, FSCK-2, RC-FSK and FSCK-3) and LBL and maximum LREs of the four approximate models for the wet oxy-fuel flames are respectively shown in Fig. 12 and Table. 3. It can be observed that, as in Case 2, FSCK-1 is in good agreement with the benchmark, especially in the high temperature region. This good accuracy is intimately related to the choice of an emission-weighted temperature as reference temperature. FSCK-2 provides the same accuracy in the high-temperature region as FSCK-1. In the low-temperature regions, it is interesting to observe that LRE of FSCK-2 is somewhat lower than those of FSCK-1 in the two small-scale wet oxy-fuel flames (Case 3a and Case 3b), while it shows opposite trends for the two large-scale wet oxy-fuel flames (Case 3c and Case 3d). The improved performance of FSCK-2 in the low-temperature region of Case $3 \mathrm{a}$ is quite different from its poor accuracy in Case 2a, even though the only difference between Case 3a and Case 2a lies in the increased mole fraction of $\mathrm{H}_{2} \mathrm{O}$. To understand the different performance of FSCK-2 in Cases 2a and 3a, the profiles of $k^{*}$ by FSCK-1 and FSCK-2 at two positions of the low-temperature region are compared in Fig. 13. It shows that the differences between the two $k^{*}$ profiles in the low-temperature regions of Case 3 by the two solution schemes are significantly reduced compared to those shown in Fig. 9 for Case 2. Clearly, this reduced difference is attributed to the presence of $\mathrm{H}_{2} \mathrm{O}$ in the low-temperature regions. In general, the cumulative k-distribution $(\mathrm{g})$ of $\mathrm{H}_{2} \mathrm{O}$ displays weaker dependence on the Planck temperature compared to $\mathrm{CO}_{2}$ and this difference may possibly be due to the fact that $\mathrm{CO}_{2}$ emits and absorbs over four distinct spectral regions.

Table 3. The maximum local relative errors of the radiative source term for Case 3 .

\begin{tabular}{|c|c|c|c|c|c|c|c|c|}
\hline \multirow{2}{*}{ Case } & \multicolumn{4}{|c|}{ High-temperature region (\%) } & \multicolumn{4}{c|}{ Low-temperature region (\%) } \\
\cline { 2 - 10 } & FSCK-1 & FSCK-2 & RC-FSK & FSCK-3 & FSCK-1 & FSCK-2 & RC-FSK & FSCK-3 \\
\hline 3a & -1.5 & -1.7 & -2.7 & -1.3 & -11.2 & -6.3 & -11.4 & -11.1 \\
\hline 3b & -3.5 & -2.7 & -4.6 & -3.4 & -8.8 & -5.8 & -8.5 & -8.7 \\
\hline 3c & -1.8 & -1.0 & -3.0 & -1.8 & -5.2 & 5.2 & -4.9 & -5.2 \\
\hline 3d & 1.9 & 2.2 & -2.2 & -1.0 & -3.7 & 7.5 & -3.4 & -3.7 \\
\hline
\end{tabular}

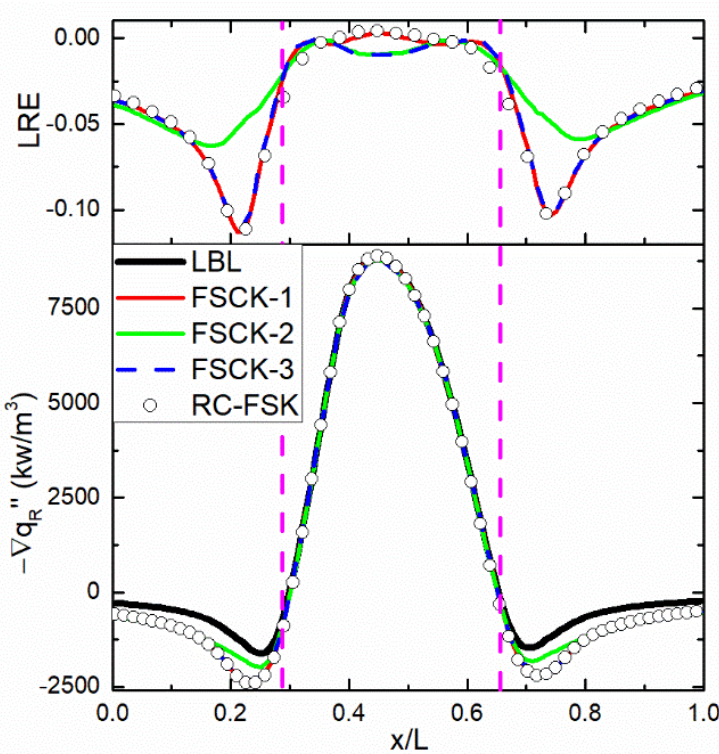

(a) $L=0.0126 \mathrm{~m}$

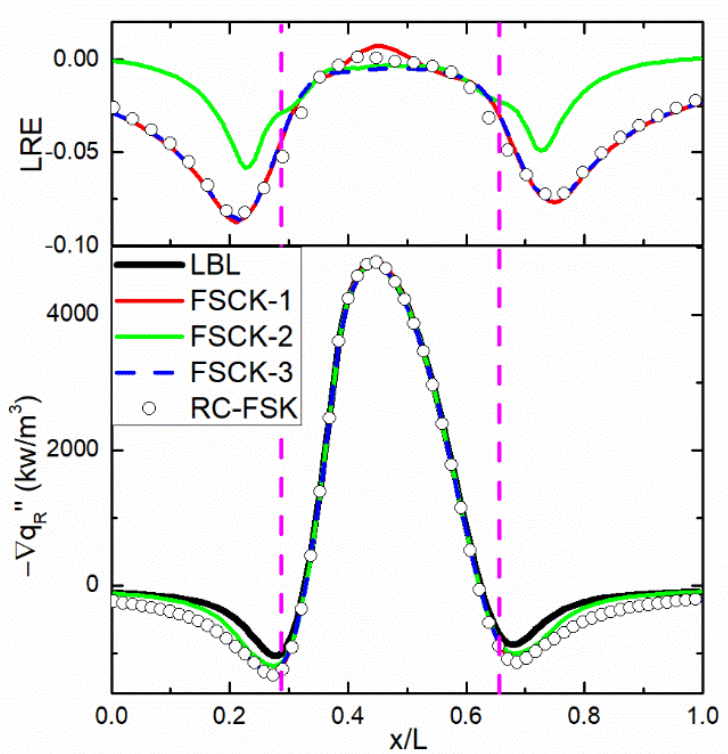

(b) $L=0.126 \mathrm{~m}$ 


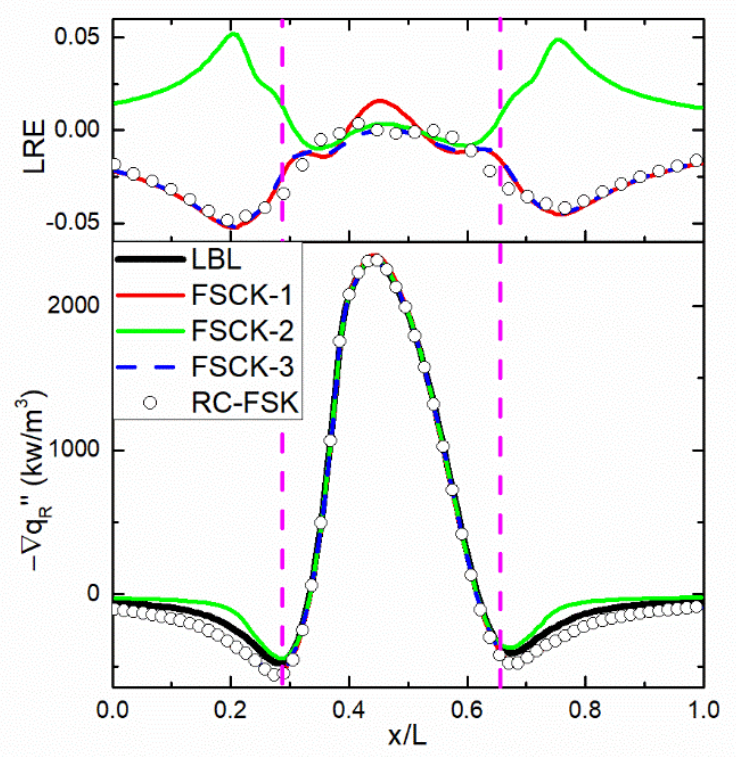

(c) $L=0.63 \mathrm{~m}$

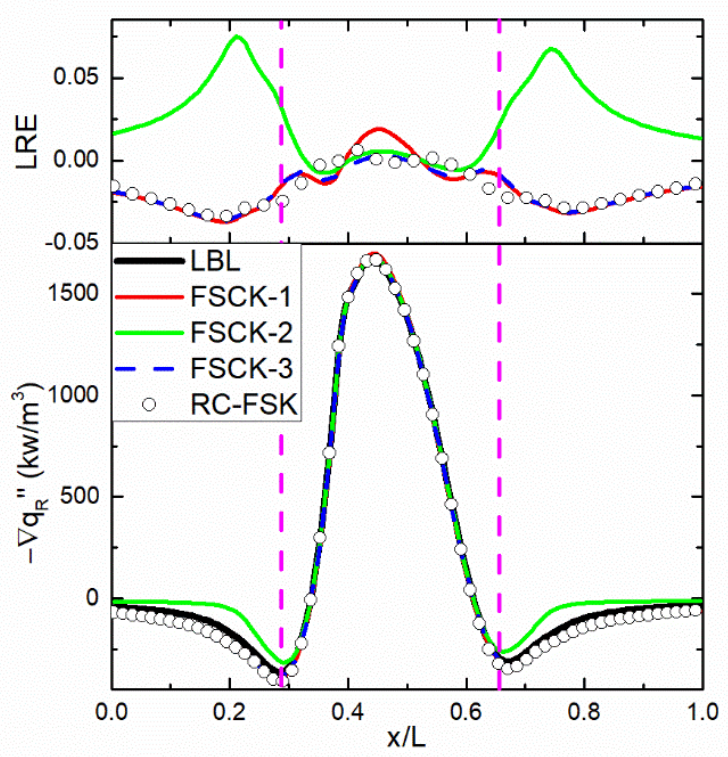

(d) $L=1.26 \mathrm{~m}$

Fig. 12 Distributions of the predicted radiative source terms and the local relative error (LREs) by FSCK-1, FSCK-2, RCFSK, FSCK-3, and LBL in the wet oxy-fuel flames (Case 3). For each diagram, the bottom frame shows the distributions of the radiative source term and the top frame shows the relative errors benchmarked by the result of LBL.

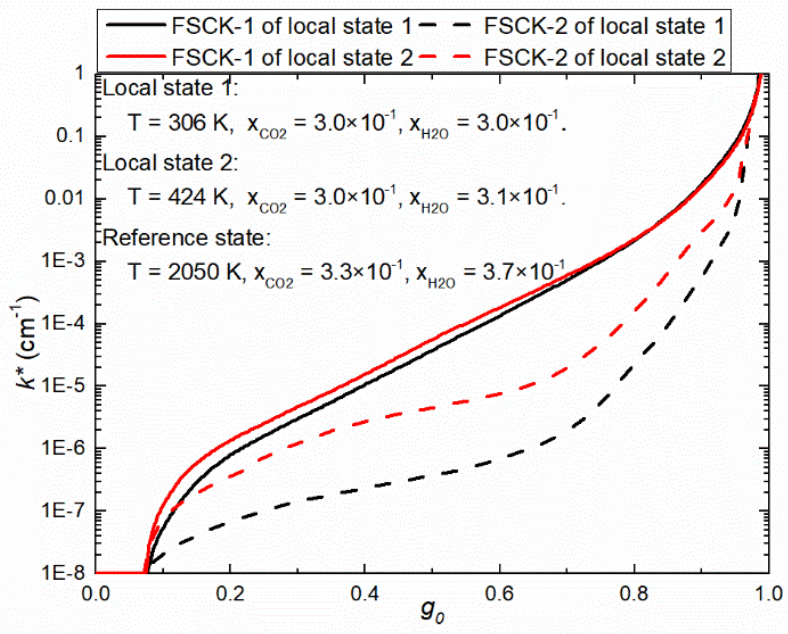

Fig. 13 The profiles of $k^{*}$ at two local states of low-temperature region of Case 3 calculated by FSCK-1 and FSCK-2.

(The reference state: $\mathrm{T}=2050 \mathrm{~K}, x_{\mathrm{CO}_{2}}=3.3 \times 10^{-1}, x_{\mathrm{H}_{2} \mathrm{O}}=3.7 \times 10^{-1}$;

local state $1: \mathrm{T}=306 \mathrm{~K}, x_{\mathrm{CO}_{2}}=3.0 \times 10^{-1}, x_{\mathrm{H}_{2} \mathrm{O}}=3.0 \times 10^{-1}$;

local state $\left.2: \mathrm{T}=424 \mathrm{~K}, x_{\mathrm{CO}_{2}}=3.0 \times 10^{-1}, x_{\mathrm{H}_{2} \mathrm{O}}=3.7 \times 10^{-1}\right)$.

As observed in Case 2 (see Fig. 8), the radiative source terms predicted by RC-FSK and FSCK-3 almost overlap with that of FSCK-1, as shown in Fig 12. In other words, FSCK-2 is more accurate than FSCK-1, FSCK-3 and RC-FSK for the two small-scale wet oxy-fuel flames (Case $3 a$ and Case $3 b$ ), while an opposite trend is observed for the two large-scale wet oxy-fuel flames (Case 3c and Case 3d).

Figure 14 displays the radiative heat fluxes of Case 3 predicted by FSCK-1, FSCK-2, FSCK-3, RC-FSK and LBL. Different from the dry oxy-fuel flame in Case $2 a$, the radiative heat flux of the smallest scale wet oxy-fuel flame, Case $3 a$, 
predicted by the four approximate methods are almost undistinguishable. For the other three sub-cases, FSCK-1, FSCK-3 and RC-FSK demonstrate the same accuracy. FSCK-2 is much more accurate than FSCK-1, FSCK-2 and FSCK-3 for Case 3b, while it predicts less accurate results in Case $3 \mathrm{c}$ and $3 \mathrm{~d}$.

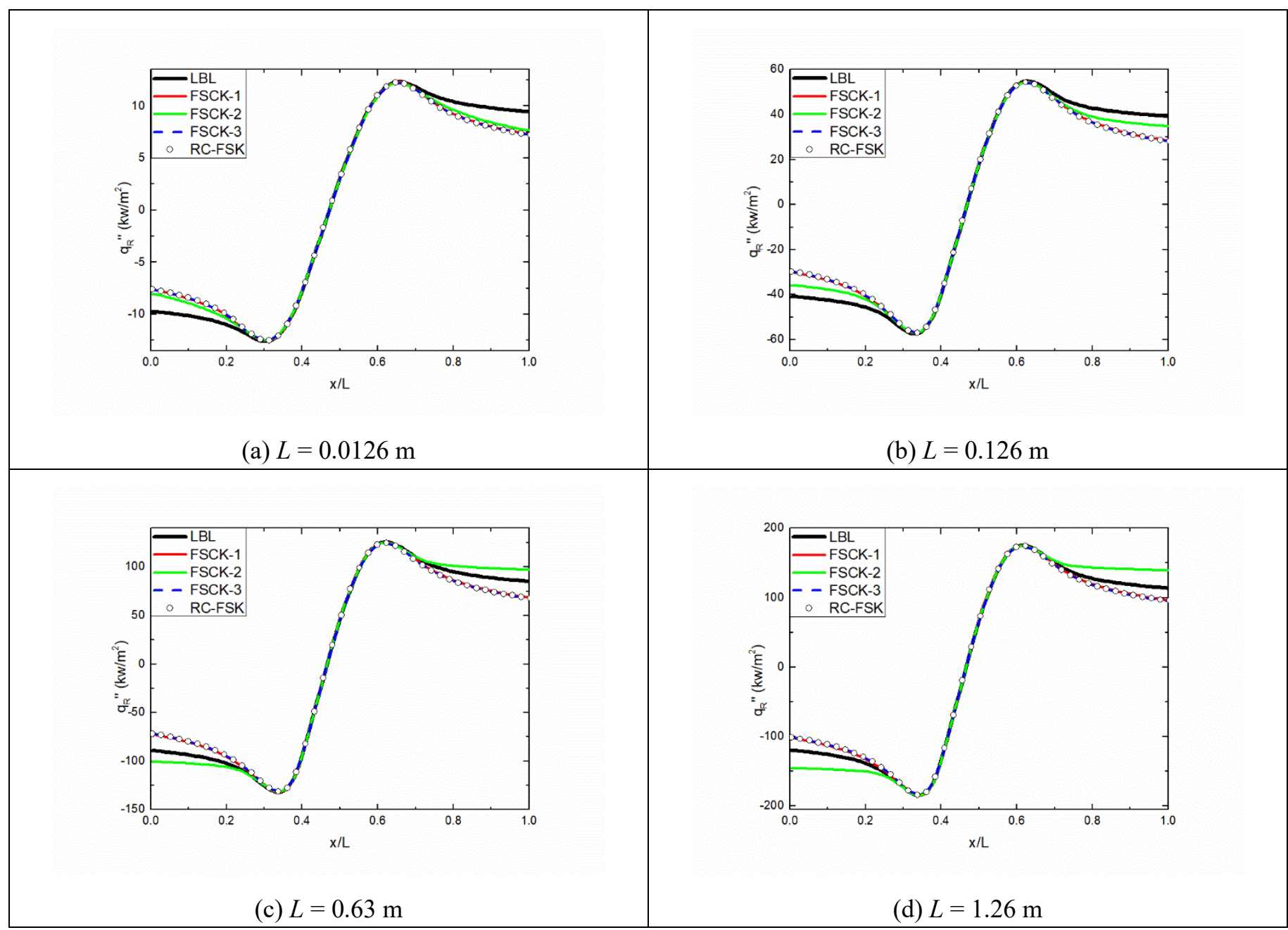

Fig. 14 Distributions of the predicted radiative heat flux by FSCK-1, FSCK-2, RC-FSK, FSCK-3, and LBL in the four wet oxy-fuel flames with different separation distances (Case 3).

In summary, the predictions by four approximate models (FSCK-1, FSCK-2, RC-FSK and FSCK-3) in the hightemperature regions of conventional fuel-air and dry and wet oxy-fuel flames agree well with the LBL solution. The local relative errors, LREs, of the four approximate models in the low-temperature regions of both the dry and wet oxy-fuel flames are larger than those of the conventional fuel-air flames, owing to the enhanced absorption due to higher concentrations of $\mathrm{CO}_{2}$ and $\mathrm{H}_{2} \mathrm{O}$.

\subsection{Effects of the reference temperature or the Planck temperature on oxy-fuel flames}

As displayed above, FSCK-1, FSCK-2 and RC-FSK predict almost the same results for conventional fuel-air and dry and wet oxy-fuel flames when the emission-weighted temperature is selected as the reference temperature. In this section, the influence of the reference temperature for FSCK-1, FSCK-2 and FSCK-3, and the Planck temperature for RC-FSK is discussed. For the convenience of discussion, four parameters are compared here, including the local relative error of radiative source term (LRE) at the position of highest temperature, the absolute relative error of radiative source term of the lowtemperature region (AEL), the total absolute relative error of radiative source term (TAE) [25], and the error of heat flux at the right wall (EF). These quantities are defined as: 
$\mathrm{AEL}=\int_{0}^{L}\left|-\boldsymbol{\nabla} \cdot \dot{q}_{R_{F S C K}}^{\prime \prime}(x)+\boldsymbol{\nabla} \cdot \dot{q}_{R_{L B L}}^{\prime \prime}(x)\right| \cdot H(1000-T) d x / \int_{0}^{L}\left|\boldsymbol{\nabla} \cdot \dot{q}_{R_{L B L}}^{\prime \prime}(x)\right| d x$

$\mathrm{TAE}=\int_{0}^{L}\left|-\boldsymbol{\nabla} \cdot \dot{q}_{R}^{\prime \prime}{ }_{F S C K}(x)+\boldsymbol{\nabla} \cdot \dot{q}_{R_{L B L}}^{\prime \prime}(x)\right| d x / \int_{0}^{L}\left|\boldsymbol{\nabla} \cdot \dot{q}_{R_{L B L}}^{\prime \prime}(x)\right| d x$

$\mathrm{EF}=\left(\dot{q}_{R F C K}^{\prime \prime}(L)-\dot{q}_{R_{L B L}}^{\prime \prime}(L)\right) / \dot{q}_{R_{L B L}}^{\prime \prime}(L)$

Figures 15 and 16 show the variations of the above four quantities, Eqs. (16) - (19), with the reference temperature for FSCK-1, FSCK-2 and FSCK-3, and the Planck temperature for RC-FSK in both the dry and wet oxy-fuel flames of the four separation distances. The top frame of each diagram shows LREs at the position of peak temperature. It can be found that the reference temperature, $T_{0}$, has a great impact on the accuracy of FSCK-1, and a higher reference temperature results in a better accuracy in both the dry and wet oxy-fuel flames. In contrast, the accuracy of FSCK-2, FSCK-3 and RC-FSK seems to be quite independent of the choice of reference temperature, $T_{0}$, or the Planck temperature, $T_{P}$.

The second frames of Figures 15 and 16 display the absolute relative errors of radiative source term of low-temperature region (AELs). In the low-temperature region, FSCK-1, FSCK-3 and RC-FSK seem to have little dependence on $T_{0}$ or $T_{P}$. FSCK-2 shows the same trend as FSCK-1, FSCK-3 and RC-FSK, except for the small-scale dry oxy-fuel flame (the second frame of Figs. 15(a) and 16(b)).

As for the total absolute errors of radiative source term (TAEs), the third frames of Fig. 15 and 16, the reference temperature has a fairly strong impact on the accuracy of FSCK-1, and a higher reference temperature results in improved accuracy of FSCK-1, which agrees with the finding of Solovjov et al. [25] As displayed above, FSCK-1 can be quite accurate when the emission-weighted temperature ( $2060 \mathrm{~K}$ for Case 2, $2050 \mathrm{~K}$ for Case 3 ) is chosen as the reference temperature [6, 7]. This demonstrates the importance of selecting an appropriate reference temperature for FSCK-1. For FSCK-2, The reference temperature (when $T_{0} \geq 800 \mathrm{~K}$ ) has little influence on the accuracy for all the dry and wet oxy-fuel flames, except for the two small-scale dry oxy-fuel flames, where the large absolute error of the low-temperature region (AEL) contributes to a large portion of the total absolute error (TAE) for Cases 2a and 2b, as shown in Figs. 15(a) and 15(b). The accuracies of FSCK-3 and RC-FSK display little dependence on the reference temperature and the Planck temperature, respectively, when $T_{0}$ and $T_{P}$ are higher than $800 \mathrm{~K}$.

The bottom frames of Fig. 15 and 16 display the error of heat flux reaching the right wall (EF). EF of FSCK-1 decreases firstly as the reference temperature increases up a certain value, then increases slowly with further increasing the reference temperature for all the flames discussed here. When the reference temperature is equal to the emission-weighted temperature, EF of FSCK-1 is the same as that of FSCK-3 and RC-FSK. EF of FSCK-2 shows a weak dependence on the reference temperature, except for the two small-scale dry oxy-fuel flames. In addition, the accuracy of FSCK-2 in the prediction of heat flux at the right wall varies with the length scale of computational domain and the mole fraction of $\mathrm{H}_{2} \mathrm{O}$. Overall, FSCK-3 and RC-FSK show little dependence on the reference temperature or the Planck temperature, though their EF tend to decrease (increase in magnitude) with increasing the reference or Planck temperature for the two large-scale wet oxy-fuel flames, as shown in Figs. 16(c) and 16(d). This may be because the correlated spectrum assumption becomes gradually less valid when the reference temperature (FSCK-3) or the Planck temperature (RC-FSK) deviates increasingly from the local low temperature near the right wall. 

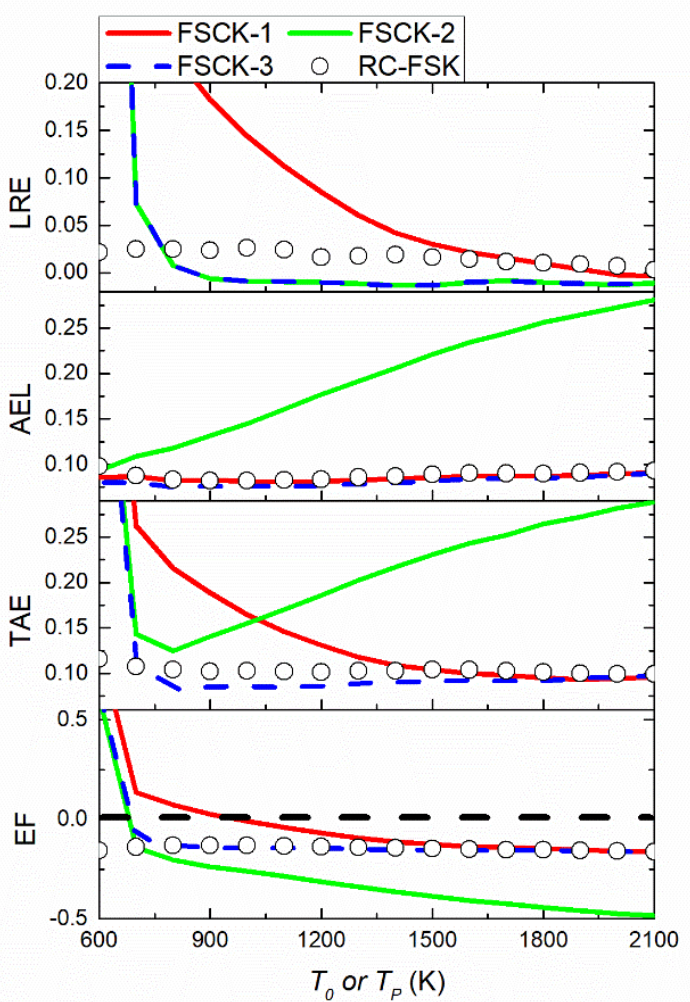

(a) $L=0.0126 \mathrm{~m}$

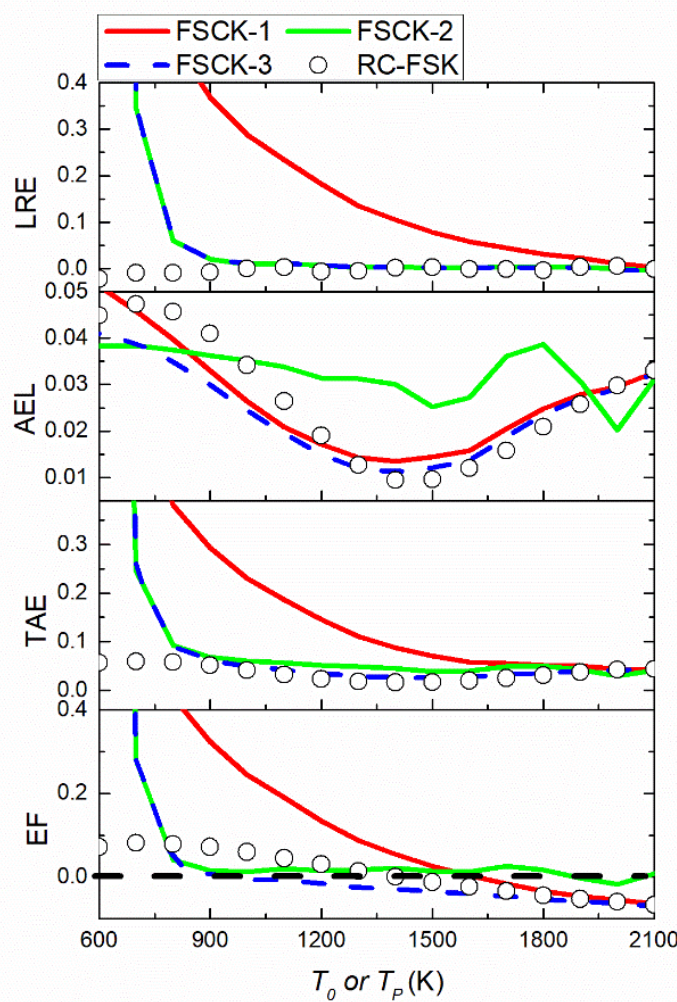

(c) $L=0.63 \mathrm{~m}$

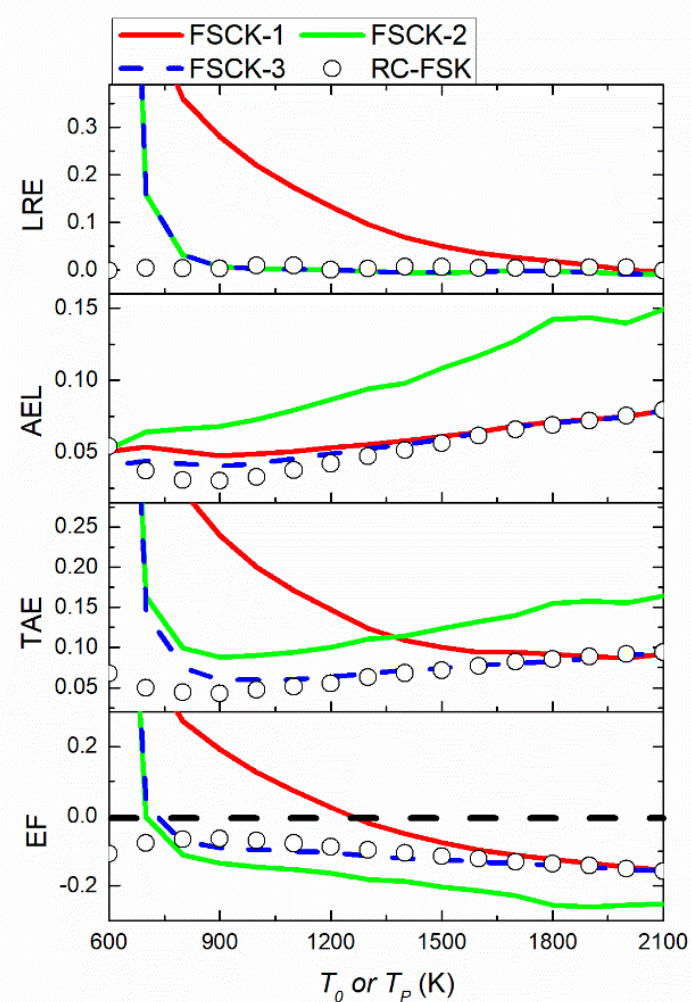

(b) $L=0.126 \mathrm{~m}$

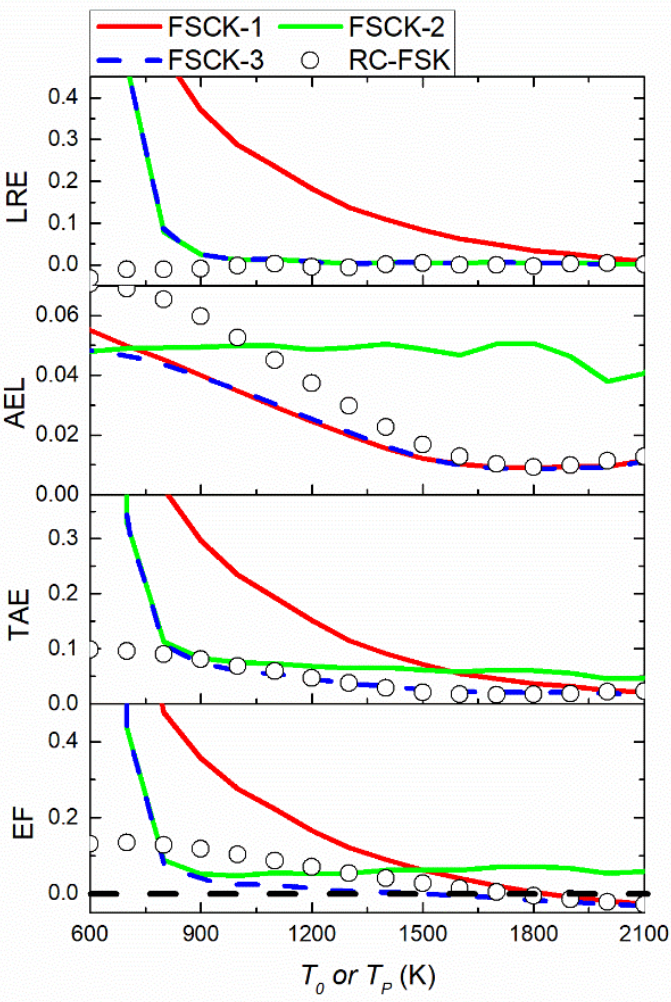

(d) $L=1.26 \mathrm{~m}$

Fig. 15 Variations of the four quantities related to the errors of the approximate models with the reference temperature for FSCK-1, FSCK-2 and FSCK-3, and the Planck temperature for RC-FSK in the dry oxy-fuel flames (Case 2). For each diagram, the top frame shows the local relative errors of radiative source term (LREs) at the position of highest temperature, the second frame shows absolute errors of radiative source term of the low-temperature region (AELs), the third frame 
shows the total absolute errors of radiative source term (TAEs), and the bottom frame shows the errors of heat flux at the right wall (EFs).

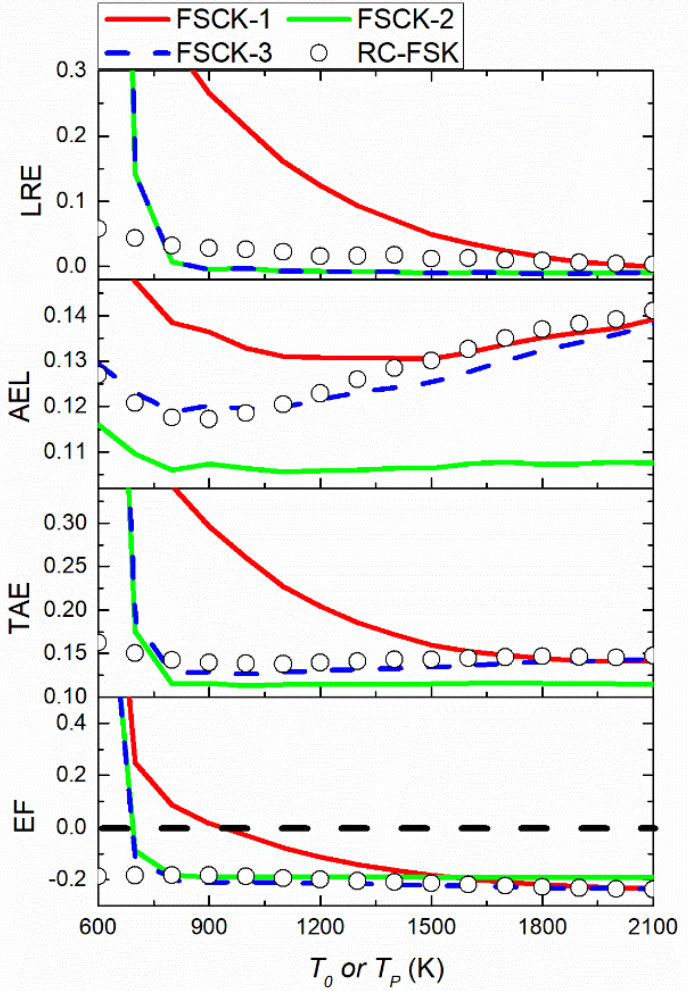

(a) $L=0.0126 \mathrm{~m}$

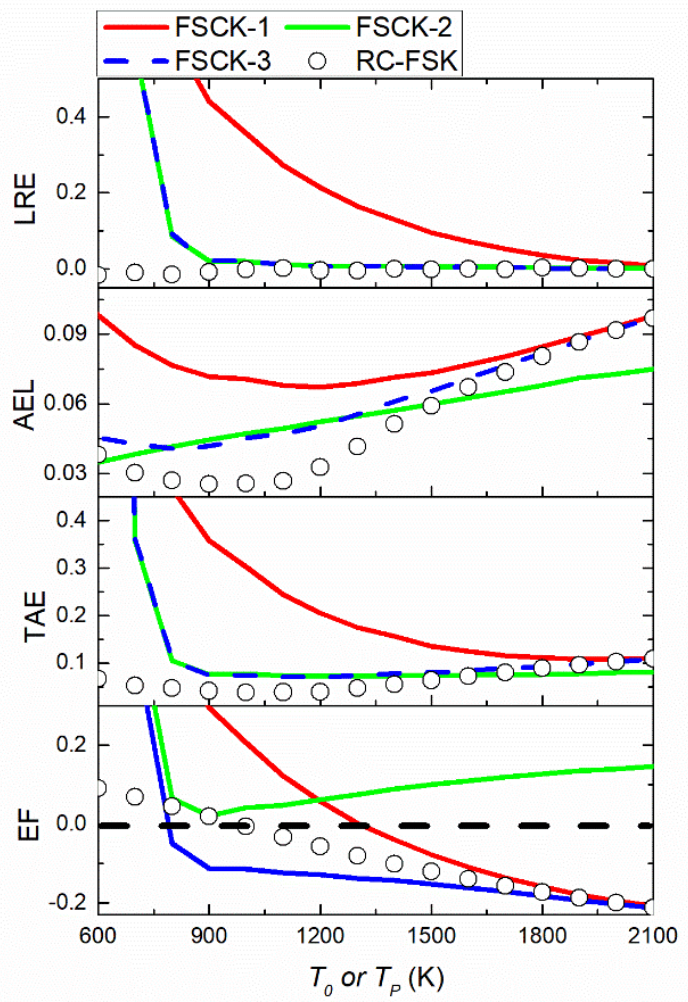

(c) $L=0.63 \mathrm{~m}$

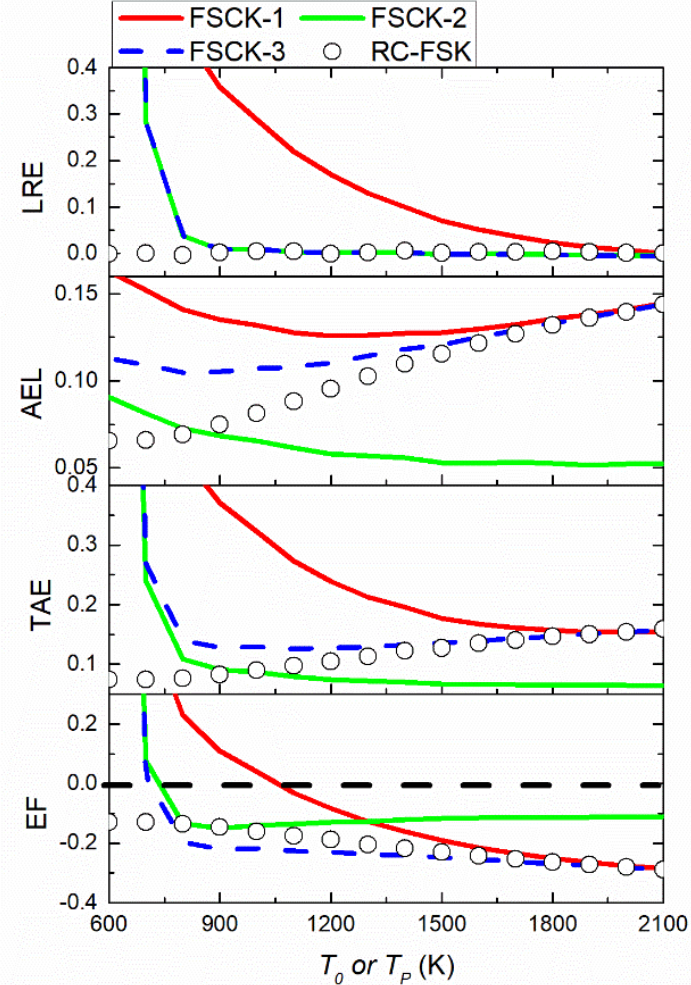

(b) $L=0.126 \mathrm{~m}$

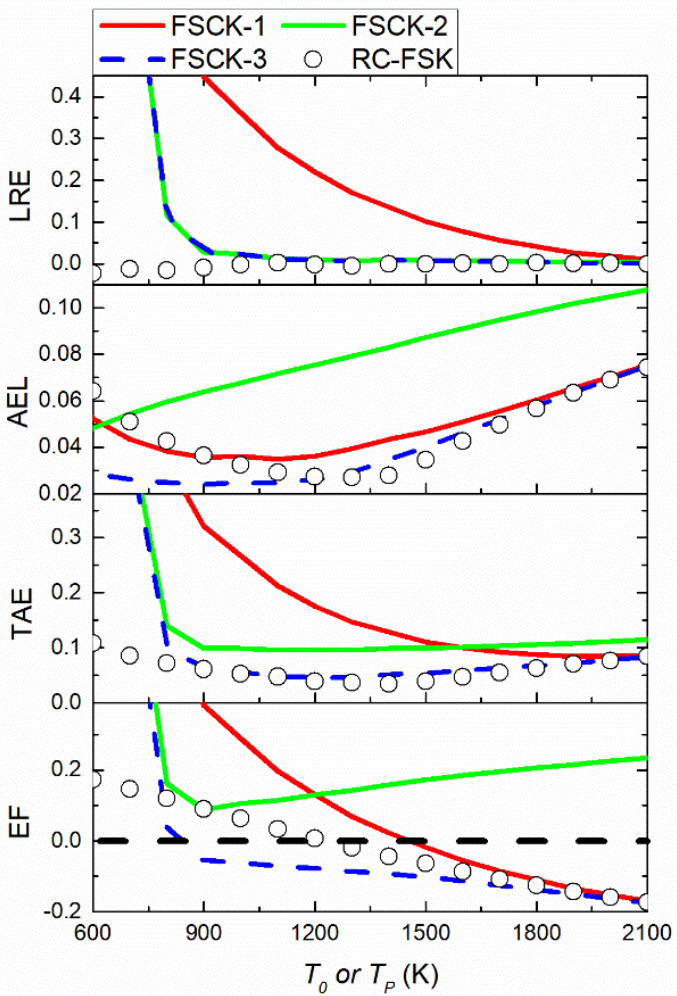

(d) $L=1.26 \mathrm{~m}$

Fig. 16 Variations of the four quantities related to the errors of the approximate models with the reference temperature 
for FSCK-1, FSCK-2 and FSCK-3, and the Planck temperature for RC-FSK in the wet oxy-fuel flames (Case 3). For each diagram, the top frame shows the local relative errors of radiative source term (LREs) at the position of highest temperature, the second frame shows absolute errors of radiative source term of the low-temperature region (AELs), the third frame shows the total absolute errors of radiative source term (TAEs), and the bottom frame shows the errors of heat flux at the right wall (EFs).

\subsection{Effects of criterion temperature on the hybrid solution scheme (FSCK-3)}

As mentioned above, the reference temperature is regarded as the criterion to determine when the solution scheme by Modest and Zhang [24] or by Cai and Modest [27] should be used in the hybrid solution scheme. In this section, the emissionweighted temperature is selected as the reference temperature, and the influence of the criterion temperature is explored. Fig. 17 displays the variation of the total absolute error (TAE) with the criterion temperature in both the dry (Fig. 17(a)) and wet (Fig. 17(b)) oxy-fuel flames. It can be seen that TAEs in the dry and wet oxy-fuel flames give different trends at very low criterion temperature below $1000 \mathrm{~K}$, and then keeps nearly constant when the criterion temperature is between about $1000 \mathrm{~K}$ and $2400 \mathrm{~K}$. Above $2400 \mathrm{~K}$, it starts to increase for large-scale dry and wet oxy-fuel flames. In conclusion, it is better to choose the criterion temperature not too far away from the reference temperature when using the FSCK-3 method, though the choice of criterion temperature seems not to be critical to the performance of FSCK-3.

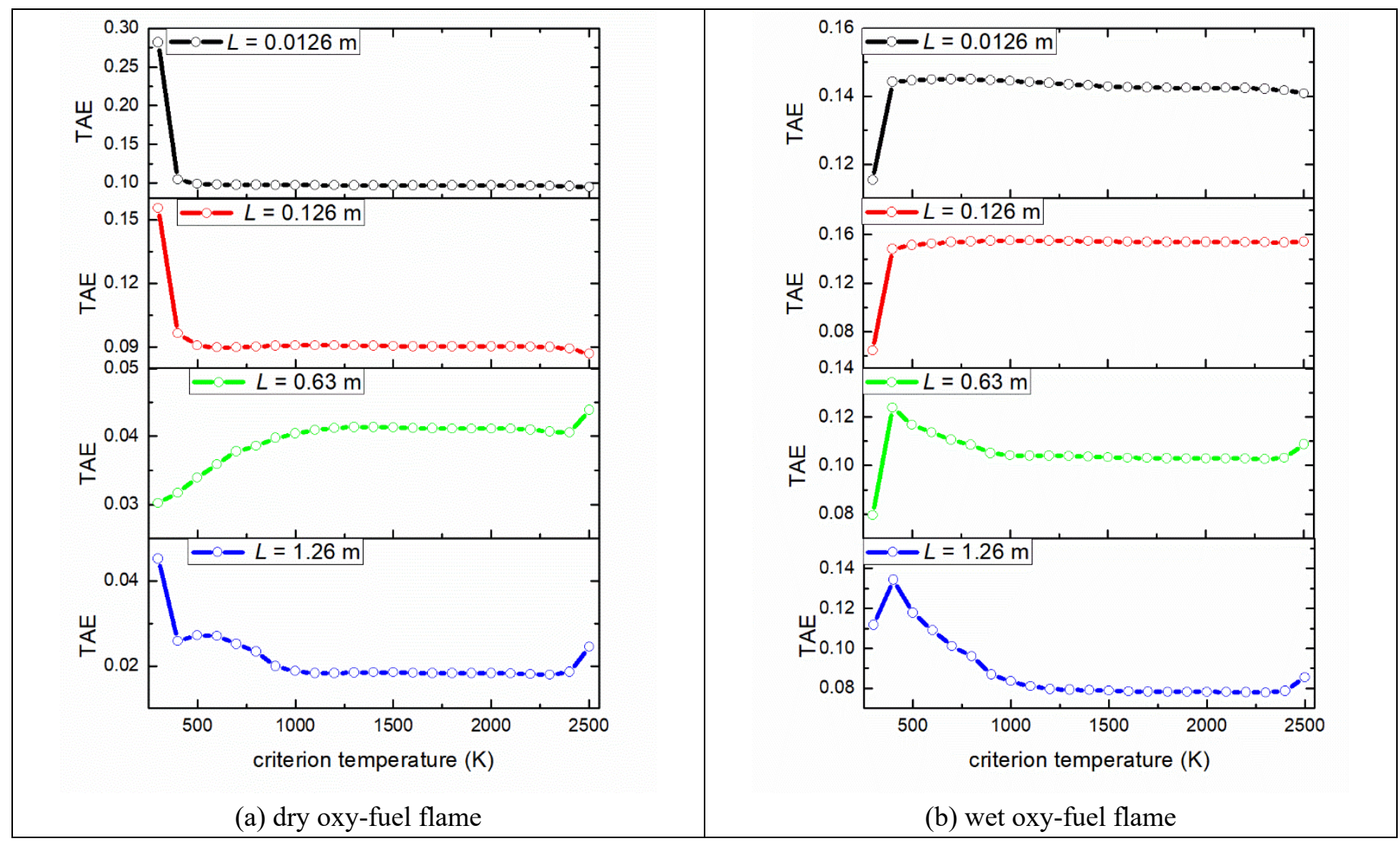

Fig. 17 Variation of the total absolute error of radiative source term (TAE) with the criterion temperature.

\section{Results and discussion of a two-dimensional dry oxy-fuel flame}

In this section, the accuracies of FSCK-1, FSCK-2, FSCK-3 and RC-FSK in a two-dimensional dry oxy-fuel flame [37] are evaluated. A combustion chamber is made of a cylinder with a size of $1000 \mathrm{~mm}$ (height) $\times 175 \mathrm{~mm}$ (radius). Methane is injected into the combustion chamber with the velocity of $4.6 \mathrm{~m} / \mathrm{s}$ through a $2.5-\mathrm{mm}$-radius nozzle which is centered in a 50 mm-radius laminar oxidizer co-flow. The oxidizer consists of $35 \% \mathrm{O}_{2}$ and $65 \% \mathrm{CO}_{2}$, and the velocity is $0.25 \mathrm{~m} / \mathrm{s}$.

The simulation is carried out by a commercial Computational Fluid Dynamics (CFD) code [38]. Figure 18 displays the schematic diagram of the $2 \mathrm{D}$ axisymmetric computational domain. The SIMPLEC algorithm is selected for velocity-pressure 
coupling, and the PRESTO and QUICK schemes are used for spatial discretization of the pressure and momentum terms [39]. The standard $\mathrm{k}-\varepsilon$ model is chosen as turbulence model, while the flamelet model with GRI Mech-3.0 [40] mechanism is selected as the combustion model. Distributions of temperature and mole fractions of $\mathrm{CO}_{2}$ and $\mathrm{H}_{2} \mathrm{O}$ are shown in Fig. 19. The peak temperature is $2293 \mathrm{~K}$.

It can be seen that the high-temperature region is located near the fuel and oxidizer inlets. To improve the resolution of the radiation calculation, part of the combustion chamber $(500 \mathrm{~mm} \times 60 \mathrm{~mm})$ is regarded as the computational domain here. Radiation calculation is conducted in a $2 \mathrm{D}$ axisymmetric cylindrical coordinates The number of grids is 50 (height) $\times 60$ (radius). DOM with the S6 angular discretization scheme is used to solve RTE, and the 32-point Gauss-Chebyshev quadrature scheme is used [32]. The emission-weighted temperature is chosen as the reference temperature.

Figure 20 shows the distribution of radiative source term predicted by the LBL method, in which the maximum radiative source term is $9220 \mathrm{kw} / \mathrm{m}^{3}$. Figure 21 exhibits the relative errors of FSCK-1, FSCK-2, FSCK-3 and RC-FSK. FSCK-1 can provide good accuracy compared with the LBL method, and the error ranges from $-1.6 \%$ to $1.0 \%$, as in the one-dimensional problems discussed above. FSCK-2 is as accurate as FSCK-1 in the high-temperature region; however, it demonstrates large errors in the low-temperature region. The error of FSCK-2 is between $-4.0 \%$ and $0.2 \%$. The error of FSCK-3 ranges from $1.6 \%$ to $0.2 \%$, which combines the advantages of FSCK-1 and FSCK-2 in the low- and high-temperature regions. The error of RC-FSK ranges from $-1.4 \%$ to $0.7 \%$, which is almost the same as that of FSCK-3. In conclusion, the performances of FSCK-1, FSCK-2, FSCK-3 and RC-FSK are the same as in 1D dry oxy-fuel flames.

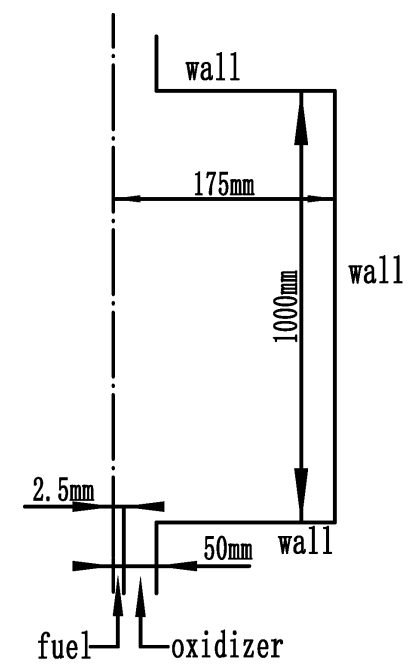

Fig. 18 Schematic diagram of the computational domain. 


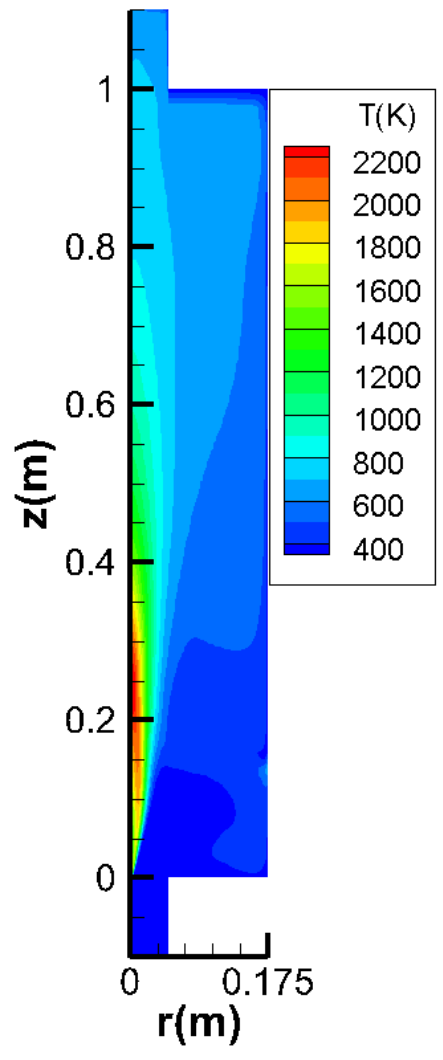

(a) Temperature (peak $2293 \mathrm{~K}$ )

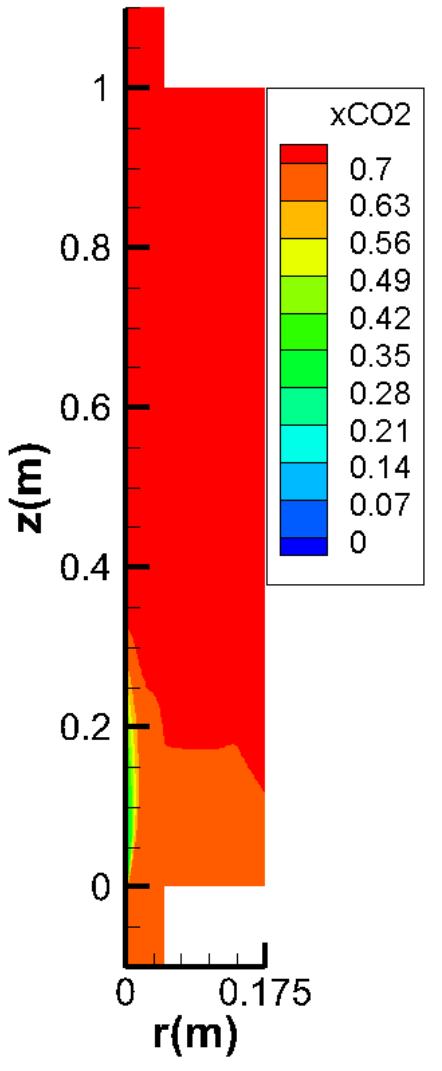

(b) $\mathrm{CO}_{2}$ mole fraction (peak 0.725)

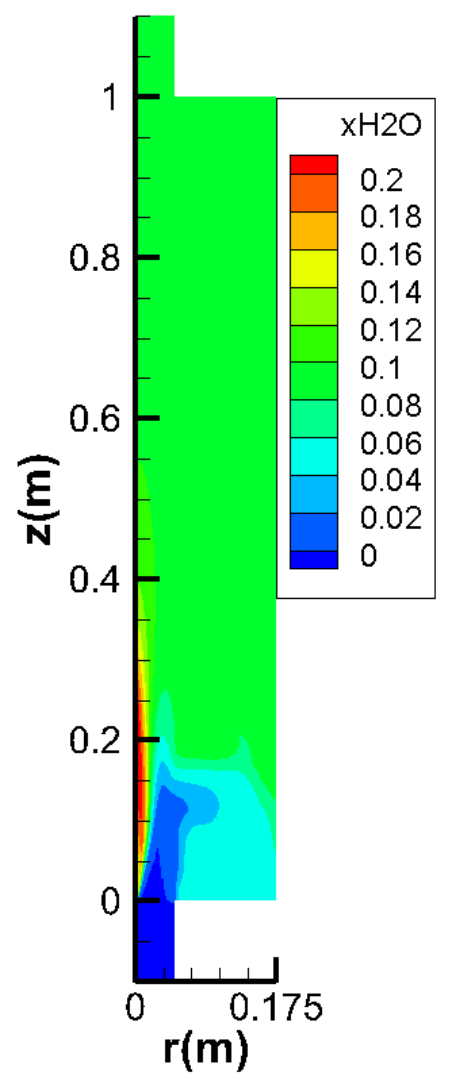

(C) $\mathrm{H}_{2} \mathrm{O}$ mole fraction (peak 0.231)

Fig. 19 Distributions of temperature and mole fractions of $\mathrm{CO}_{2}$ and $\mathrm{H}_{2} \mathrm{O}$ in the 2D dry oxy-fuel flame.

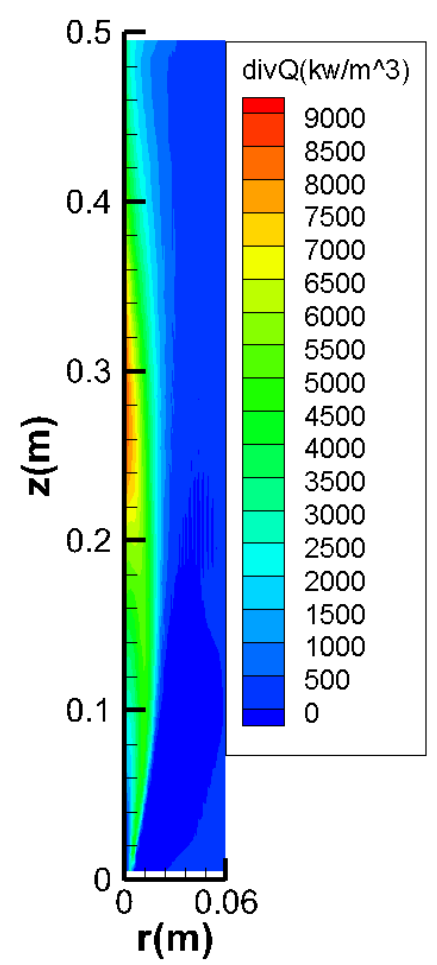

Fig. 20 Distribution of radiative source term calculated by the LBL method. 


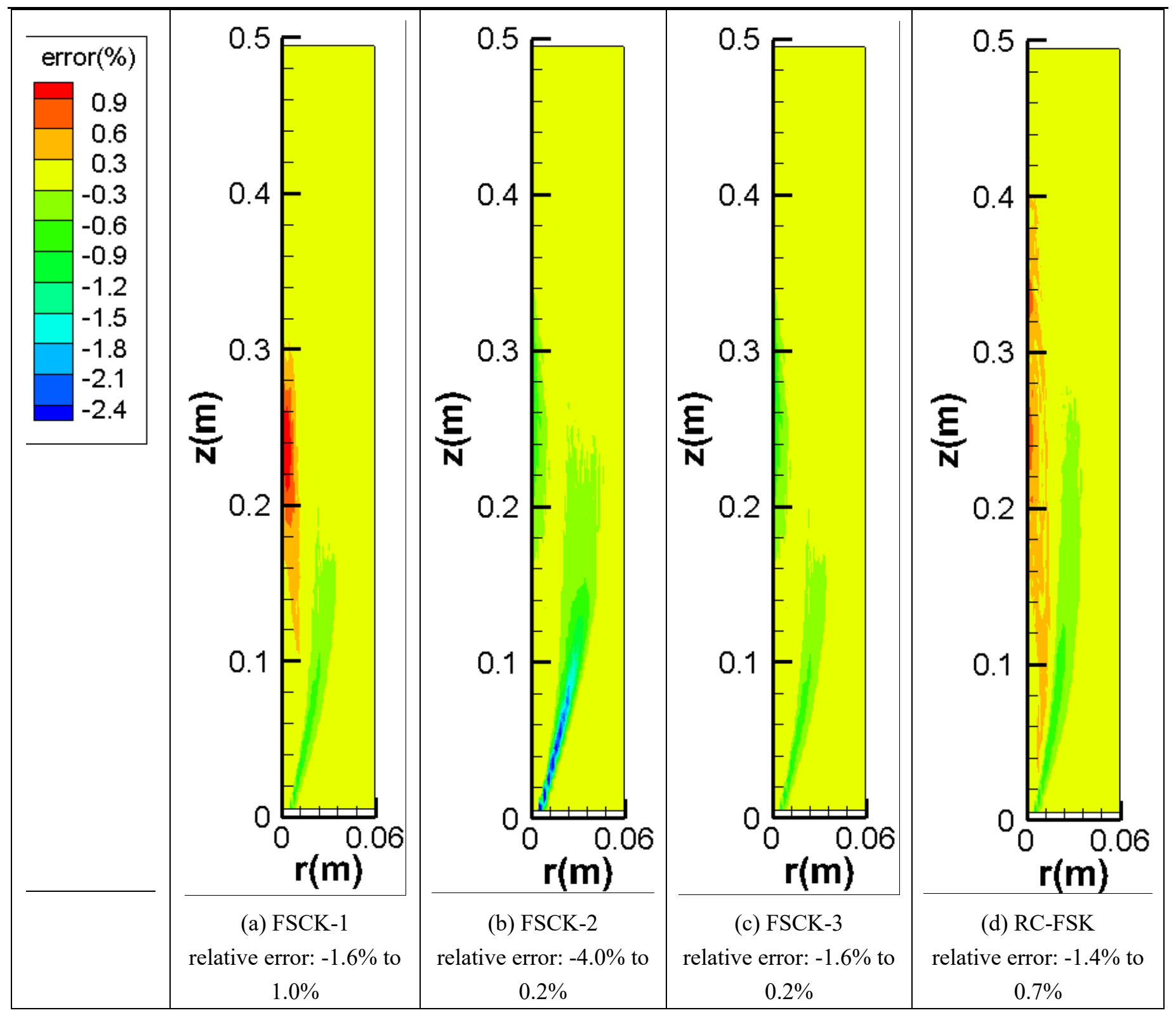

Fig. 21 Distributions of relative errors of FSCK-1, FSCK-2, FSCK-3 and RC-FSK.

\section{Conclusions}

In this work, an improved hybrid correlated-K solution scheme of FSCK was proposed and the accuracy of FSCK-1, FSCK-2, FSCK-3 and RC-FSK was evaluated in 1D conventional fuel-air flames and dry and wet oxy-fuel flames with four different separation distances, as well as in a 2D lab-scale dry oxy-fuel flame. LBL results were obtained and used as the benchmark solution. The following conclusions can be drawn from this work:

(1) FSCK-1 (Modest and Zhang, 2002 [24]) produces accurate predictions when the emission-weighted temperature is chosen as the reference temperature. However, this model is highly dependent on the reference temperature.

(2) The accuracy of FSCK-2 (Cai and Modest, 2014 [27]) shows little dependence on the reference temperature except for the two small-scale dry oxy-fuel flames. Errors are mainly concentrated in the low-temperature region, and its overall accuracy depends on the length scale of computational domain. In the low-temperature regions, FSCK-2 produces larger errors for dry oxy-fuel flames, especially the small-scale flame, and large-scale wet oxy-fuel flames in comparison with FSCK-1.

(3) The accuracy of FSCK-3 (hybrid correlated-K solution scheme proposed in this work) is also nearly unaffected by the reference temperature. FSCK-3 combines the advantages of FSCK-1 and FSCK-2 in the low- and high-temperature 
regions.

(4) The results of RC-FSK and FSCK-3 are almost the same in all the flames, when the reference temperature for FSCK3 is equal to the Planck temperature for RC-FSK.

(5) The criterion temperature of FSCK-3 to determine which correlated-K solution scheme should be used has little effects on the result of FSCK-3 over a wide range. It is recommended to set the criterion temperature to the reference temperature for simplicity and accuracy.

In conclusion, FSCK-1 can predict good results in all the 1D conventional fuel-air flames, dry and wet oxy-fuel flames and the 2D dry oxy-fuel flame when the emission-weighted temperature is selected as the reference temperature. FSCK-3 and RC-FSK can provide almost the same accuracy as FSCK-1, and the reference temperature or Planck temperature has little impacts on the accuracies.

\section{Acknowledgments}

The authors would like to thank the support of "National Natural Science Foundation of China" (Grant No. 50606004) for previously related research and the support of "Overseas Expertise Introduction Project for Discipline Innovation" (111 project, Grant No.B08009 ) for international academic exchanges.

\section{Reference}

[1] Scheffknecht G, Al-Makhadmeh L, Schnell U, Maier J. Oxy-fuel coal combustion-A review of the current state-of-theart. Int J of Greenh Gas Control 2011; 5: S16-S35.

[2] $\mathrm{Hu}$ Y, Yan J. Numerical simulation of radiation intensity of oxy-coal combustion with flue gas recirculation. Int J Greenh Gas Control 2013; 17: 473-480.

[3] Chen L, Yong S Z, Ghoniem A F. Oxy-fuel combustion of pulverized coal: Characterization, fundamentals, stabilization and CFD modeling. Progress in Energy and Combustion Science 2012; 38(2): 156-214.

[4] Edge P, Gharebaghi M, Irons R, Porter R, Porter R T J, Pourkashanian M, Smith D, Stephenson P, Williams A. Combustion modelling opportunities and challenges for oxy-coal carbon capture technology. Chem Eng Res Des 2011; 89(9): 1470-1493.

[5] Chu H, Gu M, Consalvi J L, Liu F, Zhou H. Effects of total pressure on non-grey gas radiation transfer in oxy-fuel combustion using the LBL, SNB, SNBCK, WSGG and FSCK methods. J Quant Spectros Radiat Transf 2016; 172: 2435 .

[6] Kez V, Liu F, Consalvi J L, Strohle J, Epple B. A comprehensive evaluation of different radiation models in a gas turbine combustor under conditions of oxy-fuel combustion with dry recycle. J Quant Spectros Radiat Transf 2016; 172: 121133.

[7] Kez V, Liu F, Consalvi J L, Strohle J, Epple B. Assessment of several gas radiation models for radiative heat transfer calculations in a three-dimensional oxy-fuel furnace under coal-fired conditions. Int J Therm Sci 2017; 120: $289-302$.

[8] Ströhle J. Wide band correlated-k approaches for non-grey radiation modelling in oxy-fuel combustion with dry recycling. Fuel 2011; 90(10): 3007-3013.

[9] Ströhle J. Assessment of the re-ordered wide band model for non-grey radiative transfer calculations in 3D enclosures. J Quant Spectros Radiat Transf 2008; 109(9): 1622-1640.

[10] Denison M K, Webb B W, Denison M K. A spectral line-based weighted sum-of-gray-gases model for arbitrary RTE solvers. ASME J Heat Transf 1993; 115:1004-1012.

[11] Porter R, Liu F, Pourkashanian M, Williams A, Smith D. Evaluation of solution methods for radiative heat transfer in gaseous oxy-fuel combustion environments. J Quant Spectros Radiat Transf 2010; 111(14): 2084-2094.

[12] Clements A G, Porter R, Pranzitelli A, Pourkashanian M. Evaluation of FSK models for radiative heat transfer under oxyfuel conditions. J Quant Spectros Radiat Transf 2015; 151: 67-75. 
[13] Yin C, Johansen L C, Rosendahl L A, Kær S K. New weighted sum of gray gases Model applicable to computational fluid dynamics (CFD) modeling of oxy-fuel combustion: Derivation, validation and implementation. Energ Fuel 2010; 24(12): 6275-6282.

[14] Yin C. Prediction of air-fuel and oxy-fuel combustion through a generic gas radiation property model. Appl Energ 2017; 189: 449-459.

[15] Yin C. Nongray-gas effects in modeling of large-scale oxy-fuel combustion processes. Energ Fuel 2012; 26(6): 33493356.

[16] Johansson R, Andersson K, Leckner B, Thunman H. Models for gaseous radiative heat transfer applied to oxy-fuel conditions in boilers. Int J Heat Mass 2010; 53: 220-230.

[17] Nakod P, Krishnamoorthy G, Sami M, Orsino S. A comparative evaluation of gray and non-gray radiation modeling strategies in oxy-coal combustion simulations. Appl Therm Eng 2013; 54(2): 422-432.

[18] Guo J, Li X, Huang X, Liu Z, Zheng C. A full spectrum k-distribution based weighted-sum-of-gray-gases model for oxyfuel combustion. Int J Heat Mass Transf 2015; 90: 218-226.

[19] Rajhi M A, Ben-Mansour R, Habib M A, Nemitallah M A, Andersson K. Evaluation of gas radiation models in CFD modeling of oxy-combustion. Energ Convrs Manage 2014; 81: 83-97.

[20] Zhang J, Ito T, Ito S, Riechelmann D, Fujimori T. Numerical investigation of oxy-coal combustion in a large-scale furnace: Non-gray effect of gas and role of particle radiation. Fuel 2015: 139; 87-93.

[21] Bordbar M H, Wecel G, Hyppanen T. A line by line based weighted sum of gray gases model for inhomogeneous $\mathrm{CO}_{2}-$ $\mathrm{H}_{2} \mathrm{O}$ mixture in oxy-fired combustion. Combust and flame 2014; 161(9): 2435-2445.

[22] Kangwanpongpan T, França F H, da Silva R C, Schneider P S, Krautz H J. New correlations for the weighted-sum-ofgray-gases model in oxy-fuel conditions based on HITEMP 2010 database. Int J Heat Mass Transf 2012; 55: 7419-7433.

[23] Modest M F, Riazzi R J. Assembly of full-spectrum k-distribution from a narrow-band database; effects of mixing gases, gases and nongray absorbing particles, and mixtures with nongray scatterers in nongray enclosures. J Quant Spectrosc Radiat Transf 2005; 90: 169-189.

[24] Modest M F, Zhang H. The full-spectrum correlated-k distribution for thermal radiation from molecular gas-particulate mixtures. ASME J Heat Transf 2002; 124(1): 30-38.

[25] Solovjov V P, Webb B W, Andre F. The rank correlated FSK model for prediction of gas radiation in non-uniform media, and its relationship to the rank correlated SLW model. J Quant Spectrosc Radiat Transf 2018; 214: 120-132.

[26] Maurente F, Bruno A B, França F H R, Howell J R. Non-dimensional wavenumber in full-spectrum k-distribution computations with or without a reference state. J Quant Spectros Radiat Transf 2017; 196: 222-229.

[27] Cai J, Modest M F. Improved full-spectrum k-distribution implementation for inhomogeneous media using a narrowband database. J Quant Spectros Radiat Transf 2014; 141: 65-72.

[28] Wang C, He B, Modest M F, Ren T. Efficient full-spectrum correlated-k-distribution look-up table. J Quant Spectros Radiat Transf 2018; 219: 108-116.

[29] Wang C, Ge W, Modest M F, He B. A full-spectrum k-distribution look-up table for radiative transfer in nonhomogeneous gaseous media. J Quant Spectros Radiat Transf 2016; 168: 46-56.

[30] Pierrot L, Riviere P, Soufiani A, Taine J. A fictitious-gas-based absorption distribution function global model for radiative transfer in hot gases. J Quant Spectrosc Radiat Transf 1999: 62(5): 609-624.

[31] Cheng Z, Wehrmeyer J A, Pitz R W. Experimental and numerical studies of opposed jet oxygen-enhanced methane diffusion flames. Combust Sci Technol 2006; 178(12): 2145-2163.

[32] Modest M F. Radiative heat transfer. 3rd ed. New York: Academic Press; 2013.

[33] Rothman L S, Gordon I E, Barber R J, Dothe H, Gamache R R, Goldman A, Perevalov V I, Tashkun S A, Gamache J. HITEMP, the high-temperature molecular spectroscopic database. J Quant Spectros Radiat Transf 2010; 111: 2139-2150.

[34] Kochanov R V, Gordon I E, Rothman L S, Wcislo P, Hill C, Wilzewski J S. HITRAN application programming interface (HAPI): a comprehensive approach to working with spectroscopic data. J Quant Spectrosc Radiat Transf 2016; 177: 1530. 
[35] Chu H, Liu F, Zhou H. Calculations of gas thermal radiation transfer in one-dimensional planar enclosure using LBL and SNB models. Int J Heat Mass Transf 2011; 54: 4736-4745.

[36] Consalvi J L, Liu F. Radiative heat transfer in the core of axisymmetric pool fires - I: Evaluation of approximate radiative property models. Int J Therm Sci 2014; 84: 104-117.

[37] Abdul-Sater H, Krishnamoorthy G. An assessment of radiation modeling strategies in simulations of laminar to transitional, oxy-methane, diffusion flames. Appl Them Eng 2013; 61: 507-518.

[38] ANSYS FLUENT User's Guide, Version 12, ANSYS Inc., Canonsburg, PA, 2009.

[39] Abdul-Sater H, Krishnamoorthy G, Mario D. Predicting radiative heat transfer in oxy-methane flame simulations: an examination of its sensitivities to chemistry and radiative property models. Journal of Combustion 2015; 1-20.

[40] Smith G P, Golden D M, Frenklach M, et al. GRI Mech-3.0: 1999. <http://www.me.berkeley.edu/grimech/>. 